\title{
Persée
}

http://www.persee.fr

\section{Sources pour l'étude de la protohistoire d'Hispanie. Pour une nouvelle lecture}

\author{
Marco V. Garcia Quintela \\ Dialogues d'histoire ancienne, Année 1991, Volume 17, Numéro 1 \\ p. $61-99$
}

Voir l'article en ligne

\section{Avertissement}

L'éditeur du site «PERSEE » - le Ministère de la jeunesse, de l'éducation nationale et de la recherche, Direction de l'enseignement supérieur, Sous-direction des bibliothèques et de la documentation - détient la propriété intellectuelle et les droits d'exploitation. A ce titre il est titulaire des droits d'auteur et du droit sui generis du producteur de bases de données sur ce site conformément à la loi n`98-536 du 1er juillet 1998 relative aux bases de données.

Les oeuvres reproduites sur le site «PERSEE » sont protégées par les dispositions générales du Code de la propriété intellectuelle.

Droits et devoirs des utilisateurs

Pour un usage strictement privé, la simple reproduction du contenu de ce site est libre.

Pour un usage scientifique ou pédagogique, à des fins de recherches, d'enseignement ou de communication excluant toute exploitation commerciale, la reproduction et la communication au public du contenu de ce site sont autorisées, sous réserve que celles-ci servent d'illustration, ne soient pas substantielles et ne soient pas expressément limitées (plans ou photographies). La mention Le Ministère de la jeunesse, de l'éducation nationale et de la recherche, Direction de l'enseignement supérieur, Sous-direction des bibliothèques et de la documentation sur chaque reproduction tirée du site est obligatoire ainsi que le nom de la revue et- lorsqu'ils sont indiqués - le nom de l'auteur et la référence du document reproduit.

Toute autre reproduction ou communication au public, intégrale ou substantielle du contenu de ce site, par quelque procédé que ce soit, de l'éditeur original de l'oeuvre, de l'auteur et de ses ayants droit.

La reproduction et l'exploitation des photographies et des plans, y compris à des fins commerciales, doivent être autorisés par l'éditeur du site, Le Ministère de la jeunesse, de l'éducation nationale et de la recherche, Direction de l'enseignement supérieur, Sous-direction des bibliothèques et de la documentation (voir http://www.sup.adc.education.fr/bib/ ). La source et les crédits devront toujours être mentionnés. 


\begin{tabular}{|l|l|l|l|}
\hline$D H A$ & 17,1 & 1991 & $61-99$ \\
\hline
\end{tabular}

\title{
SOURCES POUR L'ÉTUDE DE LA PROTOHISTOIRE D'HISPANIE. POUR UNE NOUVELLE LECTURE
}

\author{
Marco V. GARCIA QUINTELA \\ (Université de Santiago de Compostela)
}

Dans un travail intitulé "Les peuples indigènes et la conquête romaine d'Hispanie. Essai de critique historiographique", publié dans un numéro antérieur de cette revue 1 , j'ai montré que l'interprétation historique des peuples préromains péninsulaires et de certains aspects de la conquête d'Hispanie n'ont presque pas subi d'altérations depuis plus d'un siècle.

J'y ai décrit la situation et les raisons pour lesquelles les thèses admises à propos de ce sujet ont survécu si longtemps en suscitant un étrange et durable consensus entre les diverses tendances historiographiques. La conclusion était très négative : l'immobilisme et le consensus évoqués se sont établis à partir de trois faits (a) on a considéré la situation péninsulaire comme une entité autosuffisante et, par conséquent, on a rejeté le comparatisme ; (b) on a ignoré plus ou moins délibérément toute confrontation avec l'anthropologie sociale qui aurait permis une vision rénovatrice des sociétés indigènes et (c) on a accepté sans esprit critique non seulement les données des textes

1. DHA, $16,2,1990$, p. 181-210. 
antiques, mais aussi les interprétations des géographes et ethnographes de l'Antiquité, et même celles de n'importe quel "informateur" (indigène ou romain) peu qualifié.

A présent, nous essaierons fondamentalement de reprendre ce dernier point sans pour autant oublier les deux autres. Nous suivrons l'ordre conceptuel établi par la communis opinio en nous intéressant aux sources auxquelles celle-ci fait appel. En premier lieu, nous étudierons les données sur la relative abondance ou pauvreté de ressources chez les populations indigènes. Puis, nous examinerons les sources alléguées habituellement en faveur de l'existence d'une structure sociale fortement déséquilibrée. Ensuite, nous passerons du côté des Romains et étudierons les bases sur lesquelles est établie la différence entre les Romains méchants et cruels, dont le modèle serait Galba, et les Romains bons et positifs pour les indigènes, dont le modèle serait Gracchus et, globalement, tous ceux à qui on attribue la fondation de cités pour les conquis.

\section{Misère et richesse des peuples indigènes}

L'historiographie en vigueur insiste beaucoup sur l'importance de l'élevage. Les témoignages apportés, bien qu'hétérogènes (des coins de monnaies aux proies préférées des "bandits", en passant par les butins des Romains), appuient cette appréciation de manière satisfaisante. Néanmoins pour souligner l'importance de l'élevage comme moyen de subsistance, on en arrive à sous-estimer clairement les sources qui se réfèrent à la pratique de l'agriculture. Leur examen nous permettra de comprendre le poids et la diffusion qu'elle a réellement eus.

Tout au long des descriptions minutieuses de campagnes militaires à notre disposition, l'agriculture apparaît très souvent sous forme de champs dévastés, d'escarmouches à propos de fourrageurs ou de mesures pour empêcher le ravitaillement de cités assiégées. De plus, tout cela se produit sur des terres considérées habituellement comme des terres d'élevage 2 . Ce type de

2 Ainsi l'agriculture est attestée dans le bassin du Tage (Liv. XXI, 5, 2 ; App. $I b ., 64)$; le bassin moyen de l'Ebre (= Celtiberia : App. $I b ., 42,48$; Liv., XL, 33, 39, 47, 49); le bassin du Douro (= Vaccéens et région de Numance, App. Ib., 51, 53, 54, 55, 76, 77, 78, 79, 80, 81, 82, 87, 88, 89 ; Liv. frag. liv. 91 ; mention de la caelia, boisson faite à partir de grains de blé à Oros. 5, 7, 2-18; Flor. I, 31, 11) ; territoire vascon (= Navarre, Sall. Hist. $2,93)$. 
témoignages est aussi très abondant dans l'intérieur de la Catalogne et les Pyrénées, région de bandits montagnards. Caton y mène sa campagne de 195 lors de laquelle, face à la pénurie de ravitaillement dans son armée, il dit que la guerre se nourrit d'ellemême ${ }^{3}$.

Les terres habitées par les Lusitaniens, les Galiciens et les Cantabres remportent la palme comme zones misérables où le banditisme est endémique. Cependant celui-ci ne peut avoir pour cause le manque de ressources agricoles appropriées, car les sources le contredisent formellement. Le cas le plus clair est celui des Lusitaniens. En effet, Polybe a été le premier à offrir une description de cette région qu'il a connue personnellement 4 . Il s'agissait d'un Eden : animaux et hommes étaient prolifiques, les produits de la terre étaient incorruptibles, elle donnait trois récoltes à l'an, les aliments provenant de l'agriculture et de l'élevage étaient variés et bon marché 5 . A celui-ci s'ajoutent d'autres témoignages sur la dévastation de récoltes dans des faits de guerre 6 . D'autre part, la pratique de l'agriculture est, aussi, bien attestée dans le quart NordOuest de la Péninsule 7 et la corniche cantabrique ${ }^{8}$.

En plus de ces références à l'agriculture, il faut considérer qu'il s'agit de populations définitivement sédentaires, qui en arrivent, selon les régions, à former de grandes villes. Il est vrai que le vocabulaire employé dans les sources est imprécis ${ }^{9}$ et toujours sujet à

3. Liv. XXXIV, 9. Voir aussi les actions contre les Ilergètes et Indibilis : Liv., XXI, 61 ; XXII, 21 ; XXVIII, 24 et 32 ; XXIX, 3 ; actions de Caton qui corroborent ce qui précède : Liv., XXXIV, 16, 3-4 et XXXIV 19-21; plus avant dans des conjonctures différentes César et Afranius s'approvisionnent abondamment sur le terrain, Caes. B.C. I, 48-49; 52 ; 60, 1-2.

4. Voir P. PEDECH, La méthode historique de Polybe, Paris 1964, p. $571-573$.

5. XXXIV, 8, 4-10 (= Ath. VIII, 330 e). Cf. Pol. XXXIV, 9, 3 (= Str. III, 2, 15) sur la prospérité des Turdétains et des Celtes due à leur sociabilité et à leur caractère pacifique. Voir aussi sur la richesse lusitanienne, Str. III, 3, 5. Sur la première reconnaissance de la Lusitanie, Pol. III, 37, 11.

6. App. $I b ., 70,71,73$.

7. Just. XLIV, 3, 4-5 et 7 ; Str., III, 3, 7, consommation de zuthos fait à base de céréales fermentées; III, 4,17 et 18 , les femmes travaillaient la terre.

8. D.C., LIII, 29 ; Str., III, 4, 18.

9. Voir A. CAPABLO, El Léxico Pliniano sobre Hispania, Cesaraugusta, 63,1987, p. 49 s. 
discussion 10, mais rien n'indique l'existence de pratiques de transhumance et de nomadisme 11. C'est d'autant plus frappant si nous prenons en compte le fait que les mêmes auteurs qui se sont occupés de l'Hispanie ont su décrire les systèmes de pâturage des peuples du Moyen Orient 12. Enfin, les gisements archéologiques de cette période montrent un habitat de type sédentaire. Tout cela paraît évident, mais il peut être convenable de le rappeler lorsqu'on lit certaines pages de l'historiographie régnante.

Les témoignages indiqués jusqu'à présent ont la valeur de données brutes: ils ne s'inscrivent pas dans des contextes de description ou d'analyse d'économies et de sociétés. Au fil des campagnes et des faits militaires, les territoires agricoles restent à l'arrière, comme les décors de la guerre. Le passage de Polybe sur la Lusitanie constitue, sans doute, une exception. Il est dommage que nous ne puissions pas lire dans quel contexte il s'inscrivait; en tout cas, bien qu'on reconnaisse qu'il exagère les aspects paradisiaques, c'est un témoignage qu'on ne peut pas éluder et qui contredit formellement le contenu et le sens des autres textes débattus. Or, la plupart de ceux-ci ne fournissent pas de descriptions; il s'agit d'analyses élaborées par les auteurs antiques eux-mêmes et que les modernes se bornent à faire leurs. Ces passages transmettent l'idéologie des Anciens, leurs modèles explicatifs, et non pas le résultat de leurs observations.

C'est le cas d'un passage d'Appien $(I b ., 54)$. On peut apprécier son fond purement ethnocentrique avec tant de clarté qu'il n'est pas légitime d'avoir recours à ce texte en appui d'une évaluation de l'économie de la meseta. En l'an 151 Lucullus assiégeait Intercatia, dans le bassin du Douro. Les Romains ne se sentaient pas bien à cause de la peur, du sommeil et "parce qu'ils n'étaient pas habitués à la

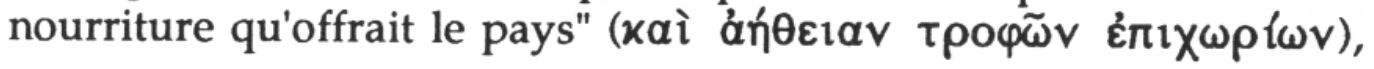

10. Comme le montre la dispute terminologique de Poséidonios contre Polybe reprise par Str. III, 4, 13.

11. Voir I. DAVIDSON, Transhumance, Spain and ethnoarchaeology, Antiquity, 54, 211, 1980, p. 144-147 avec bibliographie, qui reflète bien les difficultés du sujet. Le témoignage de César, BG., I, 51, sur les Gaulois qui viennent l'aider, est unique et contradictoire : il insiste sur leur composition bigarrée et le manque de chefs; mais les partisans de Pompée les identifient comme des ennemis et les attaquent; pour leur part les Gaulois agissent comme une troupe efficace.

12. Voir P. BRIANT, État et pasteurs au Moyen-Orient ancien, ParisCambridge, 1982, passim. 
car, poursuit Appien, ils n'avaient pas de vin, ni de sel, ni de vinaigre, ni d'huile, mais ils vivaient de blé et d'orge et de gibier et de viande de lapin cuite sans sel, ce qui provoqua la dysenterie dont beaucoup moururent. Ainsi l'auteur se borne à refléter une appréciation subjective de la réalité ; l'analyse est faite du point de vue d'une culture étrangère à la culture indigène. Si elle témoigne de quelque chose c'est de l'existence de moyens de subsistance variés : céréales et gibier qui, simplement, ne plaisaient pas aux soldats romains et encore moins au cours d'une campagne dure et incertaine.

On peut presque dire la même chose de deux textes de Strabon, cités abondamment en appui des thèses en vigueur. En effet, bien qu'il décrive la richesse du pays situé entre le Tage et le quart Nord-Ouest de la Péninsule 13, le géographe d'Amasya signale que le banditisme a été substitué aux pratiques agricoles à cause de la pauvreté des ressources des montagnards 14 . Mais le passage explique la genèse d'un phénomène, elle ne le décrit pas. Il n'y a pas de raison pour que l'ethnologie moderne accepte sans la critiquer cette explication, fruit de l'ethnologie antique : la mention du manque de ressources des montagnards comme cause du banditisme est une simple hypothèse dont la véracité reste à démontrer. Plus encore, les travaux de J.M. Vázquez Varela lui enlèvent toute vraisemblance 15.

La même situation s'apprécie dans l'analyse que Strabon fait du système alimentaire des peuples du Nord 16 : il s'agit, une fois de

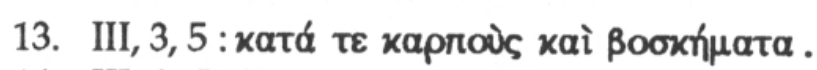

14. III, 3,5 , fin du paragraphe.

15. Voir J.M. VAZQUEZ, VARELA, Dieta Real y Dieta Imaginaria, in : J.C. BERMEJO BARRERA, Mitología y Mitos de la Hispania Prerromana II, Madrid 1986, p. 231-40 ; idem., La Alimentacion y la Cocina en la Cultura Castreña de Galicia, Estudia Zamorensia, 4, 1983, p. $392-98$; idem., et J.M. AIRA RODRIGUEZ, La Economia de los Pueblos Prerromanos del Norte de Hispania según los Testimonios Palinológicos in: G. PEREIRA MENAUT (ed.), Actas 1er Congreso Peninsular de Historia Antigua, Saint Jacques de Compostelle 1988, vol., II, p. 291-98. Voir aussi pour un âge du bronze galicien tout à fait agricole : F. CRIADO, M.J. AIRA RODRIGUEZ et F. DIAZ-FIERROS VIQUEIRA, La Construcción del Paisaje: Megalitismo y Ecología. Sierra de Barbanza, Saint Jacques de Compostelle 1986.

16. III, 3, 7. Cf. M.V. GARCIA QUINTELA, Algunhas consideracions au redor das fontes etnograficas do libro III da Xeografia de Estrabon, in: I Coloquio de Antropoloxia de Galicia, Santiago de Compostela 4-6 Febreiro 1982, La Corogne 1984, p. 15-29. L'essentiel de cette analyse a été repris par Bermejo Barrera dans Mitología y Mitos II, p. 23-24. 
plus, d'une reconstruction idéologique et non pas d'une description. Son schéma est constitué depuis Xénophon 17 et il a été démontré qu'il répond seulement de forme partielle 18 à la réalité. D'autre part, nous savons que Rome réduisait la production d'aliments "civilisés" en terre conquise, sans le moindre égard pour l'ethos de ses sujets, si elle y trouvait un avantage 19 . Enfin, à propos de ce passage nous pouvons apprécier en détail l'un des modus operandi de l'historiographie "officielle". En effet, aussi bien J. Caro Baroja que J.M. Blázquez 20 affirment que ce texte témoigne du fait que les

17. An. V, 4, 27-29, il s'agit de la description du système alimentaire des Mosynoeci. Comme les Lusitaniens ils ont des relations difficiles avec Déméter, ils s'alimentent de châtaignes au lieu de pain ; avec Athéna, ils consomment de la graisse de dauphin à la place de l'huile et avec Dionysos, ils boivent le vin sans le mélanger. Xénophon conclut son

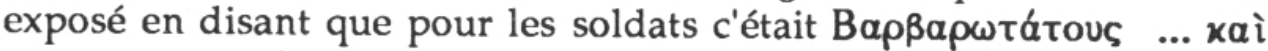

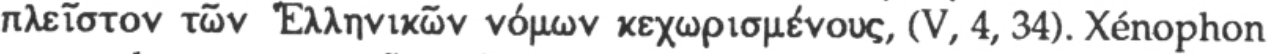
est seulement un reflet plus ancien d'une tradition ethnographique continue. Ces thèmes sont présents depuis l'Odyssée, cf. P. VIDALNAQUET, El Cazador Negro, Barcelone 1983, (Paris 1981) p. 33-61. On peut voir aussi J. PIGEAUD, La maladie de l'âme. Étude sur la relation de l'âme et $d u$ corps dans la tradition médico-philosophique antique, Paris 1981, p. 60-64 sur les théories médicales qui mettaient en relation l'alimentation avec l'ethos des individus. Bien que Strabon ne soit pas lié à ces traditions, les idées qu'il manie sont assez répandues pour qu'il puisse les employer librement ou, même, inconsciemment.

18. Cf. supra n. 15. Tous les aliments décrits par Strabon sont attestés archéologiquement (à part la vigne), mais il y a aussi des restes de beaucoup d'autres qu'il ne mentionne pas, ni aucune autre source écrite.

19. Voir A. AYMARD, L'interdiction des plantations de vignes en Gaule transalpine sous la République Romaine, in:Études d'histoire Ancienne, Paris 1967, p. 585-600 (= Mélanges Foucher, Toulouse 1948, 27-47), étude de Cic. Rep. III, 9, 16 où il examine une loi romaine qui interdit la plantation de vigne et d'oliviers en Provence à une date postérieure à 129 av. J.-C. Dans ce cas il s'agissait de renforcer l'agriculture italienne en limitant la concurrence. C'est une preuve évidente du caractère idéologique de la considération d'un peuple comme barbare en fonction de son ignorance des cultures constituant la triade méditerranéenne, car si c'est nécessaire on l'oublie complètement. D'autre part, le contexte du passage de Cicéron a un goût clairement "relativiste". 
Galiciens consommaient de la "graisse de porc". Or, le terme employé par Strabon est ßoútupov, composé de ßoũ s "vache", "bœuf" et de tupós "fromage de vache". Donc, la traduction "graisse de porc" ne peut pas être soutenue 21 , même si elle cadre mieux avec les préjugés méthodologiques des modernes qui se bornent à prolonger, en les amplifiant, les partis pris de Strabon.

Voyons un dernier témoignage communément allégué en faveur des bases économiques du brigandage. Il s'agit d'un texte connu dans lequel Diodore relate le mode de recrutement des bandits, spécialement des Lusitaniens : ce sont des jeunes dans la plénitude de leur vigueur physique et de leur audace mais pauvres, oi $\mu \alpha \lambda_{1} \sigma \tau \alpha$

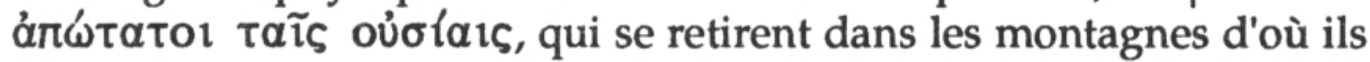
descendent pour piller et combattre les Romains 22. Dans la perspective utilitariste régnante, incapable de laisser un espace propre à la connaissance ethnologique, ce texte ne requiert aucun commentaire particulier. Néanmoins, il peut être utile de le mettre en relation avec un autre passage se référant, cette fois, à un grand héros romain. En effet, un aspect de l'action de Coriolan évoque une situation semblable à cette coutume lusitanienne. Une des accusations qu'on porte contre lui est celle d'avoir disposé du butin de guerre d'un mode contraire à la loi : au lieu de le céder au questeur pour sa vente et le dépôt du montant réuni au trésor public, Coriolan le distribua librement entre ses amis 23 . Mais ceux-ci n'étaient rien d'autre que son armée : recrutée de manière particulière parmi ses clients, ses amis, les citoyens désireux de suivre un chef chanceux et ceux qui aimaient

20. J. CARO BAROJA, Los Pueblos de España, Madrid $1981^{3}$, vol. 1,p. 348 ; J.M. BLAZQUEZ, Economía de la Hispania Romana, Bilbao 1978, p. 73.

21. Voir P. CHANTRAINE, Dictionnaire étymologique de la langue grecque, I, Paris 1968, p. 191, s.v. ßo ṽs; H. FRISK, Griechisches Etymologisches Wörterbuch, I, Heidelberg 1973, p. 261, s.v. ßoútupov .

22. V, 34, 6-7. Voir J.A. FERNANDEZ CANOSA, As maneiras de combate na Lusitania, Trab. Antrop. e Etnol. 27, 1987, p. 149-158 et la première partie de mon travail: Viriathe, chef Lusitanien, et l'idéologie trifonctionnelle indoeuropéenne, Ogam, Études Indo-Européennes 37-38, 1985-1986, sous presse.

23. D.H. Ant.Rom., VII, 62, 3-4, cf. Plut., Cor., 20, 5 ; G. DUMEZIL, Mythe et épopée III, Paris $1981^{3}$, p. 239-262. Cf. D.S. XXXIII, 1, 3 et 5 , sur le partage du butin par Viriathe. 
la guerre 24 . La seule différence appréciable entre les usages lusitaniens et la pratique de Coriolan provient de l'ethnocentrisme qui préside à la description des premiers. Quand les Lusitaniens agissent à cause de la pauvreté, les Romains le font par amour de la

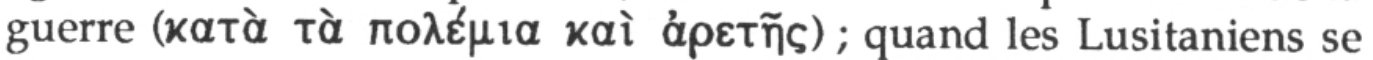
bornent à voler, les Romains distribuent de manière irrégulière le butin qu'ils ont "obtenu"; quand Rome se charge d'une action répressive de caractère militaire, dans le passage sur Coriolan Rome lui fait un procès pour punir le délit. Dans les deux cas les faits sont identiques, seul le point de vue selon lequel nos sources nous les transmettent change, ainsi que l'ethnie et le "prestige" de leurs protagonistes respectifs. Finalement, ce qui reste du texte de Diodore c'est une description, incomplète, des institutions initiatiques des guerriers lusitaniens, et non pas une description des bases économiques de la société lusitanienne 25.

Malheureusement, pour les Lusitaniens nous ne disposons pas de sources bien établies, comme c'est le cas pour les Gaulois et les Germains. En effet, nous pouvons essayer d'imaginer les interprétations modernes si, sur les premiers, nous disposions exclusivement du texte de Cicéron, selon lequel ils considéraient que le travail de la terre était déshonorant et préféraient aller en armes moissonner les champs des autres peuples (Rep. III, 9, 15) : il y aurait de puissants courants historiographiques du type de ceux qui sont actuellement en vigueur pour l'Hispanie. Heureusement, pour ce qui concerne les Gaulois, le rapport socio-anthropologique complexe de César empêche d'en arriver à ce genre de conclusions précipitées ( $B G$. VI, 13 et 15). Quoi qu'il en soit, l'interaction entre les pratiques institutionnelles, les réalités économiques et les croyances religieuses peut être très complexe et exclure toute interprétation simpliste. En ce sens, G. Dumézil nous rappelle que l'agriculture est absente du panthéon celte parce qu'elle est l'affaire d'êtres inférieurs 26 , il faudrait étudier jusqu'à quel point il a pu exister des conceptions

24. D.H. Ant.Rom., VII, 64.

25. J. MALUQUER DE MOTES, Pueblos Celtas, In: Historia de España Menéndez Pidal, tome I, vol. III : Etnología de los Pueblos de Hispania, Madrid 1954, p. 3-194, p. 153 il comprenait déjà ce texte comme le témoignage d'une institution initiatique et critiquait A. Garcia y Bellido : il n'eut aucun écho.

26. Voir G. DUMEZIL, L'oubli de l'homme et l'honneur des dieux et autres essais, Paris 1985, p. 101 et 205. La Troisième Fonction était présente à travers la métallurgie, l'artisanat et l'abondance. 
semblables chez les Lusitaniens 27. Le cas des Germains est analogue à celui des Gaulois, la description que fait César de leur coutume de brigandage nous permet de la comprendre immédiatement comme la conséquence d'un usage institutionnalisé, non pas d'une situation économique quelconque 28 .

L'ensemble de ces parallèles nous oblige à rejeter l'explication de l'origine du banditisme donnée par Diodore. Non seulement elle est ethnocentrique, en définitive le texte de César l'est aussi, mais elle est substantiellement incomplète et partielle. Elle ne reflète aucune tentative de compréhension en profondeur et par conséquent, on ne doit pas lui accorder une grande valeur.

Enfin, après une lecture des sources sans aucun type de préjugé, repassons ce qu'il nous reste des territoires minés par la pauvreté. En premier lieu, nous sommes devant des paysages qui peuvent fournir des moyens de subsistance bien diversifiés : agricoles et d'élevage (avec ce que cela suppose comme possibilité de jouir d'une alimentation suffisante et équilibrée 29 ). En second lieu, l'exploitation de ces ressources se fait à partir de noyaux de résidence plus ou moins grands mais toujours stables. Pour finir, les témoignages antiques qui évoquent les insuffisances de la base économique sont, dans chaque cas, des analyses qui reflètent le paradigme intellectuel de l'ethnographie antique, et non pas des descriptions, et ils sont idéologiquement transparents : ils signalent le territoire de la conquête 30 .

27. Selon B. GARCIA FERNANDEZ-ALBALAT, Guerra y Religión en las Gallaecia y Lusitania Antiguas, Sada, La Corogne 1990, passim, il semble que oui.

28. BG. VI, 23, 6 : Latrocinia nullam habent infamiam, quae extra fines cuiusque ciuitatis fiunt, atque ea iuuentutis exercendae ac desidiae minuendae causa fieri praedicant. Suit la description d'un recrutement "à la manière de Coriolan", BG. VI, 22, 1 et 23.

29. Dans le cas du Nord-Ouest, la consommation de poissons et crustacés est bien attestée archéologiquement, voir les travaux de VAZQUEZ VARELA déjà cités.

30. Voir sur les conquistadores espagnols d'Amérique, R. BASTIDE, El Projimo y el Extraño. El Encuentro de las Civilizaciones, Buenos Aires 1973 (Paris 1970) p. 22-3. Ce même point de vue est très clair chez Varron, $R R$. I, 16, 2, qui établit les conditions générales de la région appropriée pour implanter une exploitation agricole; il considère les conditions de voisinage, de communication, de sécurité, car "il y a beaucoup de terres excellentes qu'on n'a aucun intérêt à cultiver, à cause du brigandage des voisins (propter latrocinia vicinorum), par 
Ceci ne doit pas nous surprendre. L'anthropologie moderne nous enseigne que, dans les sociétés et les conditions les plús variées, il existe une capacité notable pour obtenir de l'environnement naturel les ressources suffisantes à leur subsistance sans effort excessif 31 . Il serait étonnant que tout le monde péninsulaire antérieur aux Romains constituât une exception. Cependant, nous devons reconnaître, pour terminer, que les conditions concrètes du processus de conquête ont dû supposer une altération sérieuse des bases économiques de ces sociétés: chaque campagne laissait derrière elle ses séquelles de destruction et de mort. Mais ceci est un effet de la lutte de Rome contre les "bandits", ce n'est pas la cause de leur existence 32.

\section{Le partage des richesses : les déséquilibres sociaux}

Il peut y avoir dans un territoire déterminé des ressources suffisantes pour le maintien d'un certain niveau de population dans des conditions appropriées, mais elles peuvent être mal réparties. L'historiographie "officielle" présente certains témoignages en faveur de l'existence de grands déséquilibres sociaux dans les populations péninsulaires qui sont, une fois de plus, la conséquence d'une interprétation discutable des textes.

Le premier de ceux-ci est l'analyse de la structure sociale gauloise faite par César (BG. VI, 13-15), qui est prise en compte de manière implicite depuis J. Costa (bien qu'il soit le seul à le citer

exemple certaines terres de Sardaigne qui sont près des Oelies, et en Espagne près de la Lusitanie". Puis, une fois pacifiées, la colonisation est viable. Voir aussi C. DOMERGUE, Introduction à l'étude des mines d'or du Nord-Ouest de la Péninsule Ibérique dans l'Antiquité, in: Legio VII Gemina, Leon 1970, p. 253-286, où il souligne que la description de Pline NH, XXXIII, 78, contient beaucoup de termes de la technique minière non latins et reflète ainsi l'existence d'une technologie préromaine complexe, p. 264-267. Ceci met en valeur à la fois la diversité de l'économie et le caractère de territoire de conquête pour les Romains.

31. C'est la thèse fondamentale du livre de M. SAHLINS, Economía de la Edad de Piedra, Madrid 1977 (Chicago 1974). Rappelons, néanmoins, que la moindre altération de l'écosystème peut avoir des conséquences catastrophiques.

32. Sur ces questions on peut toujours lire l'article d'O. LATTIMORE, La Civilisation mère de Barbarie ?, Annales ESC 17, 1962, p. 95-108. 
expressément 33 ). Il me semble que le principal problème de cette approche provient du fait qu'elle est réalisée sans une critique suffisante. En effet, César se concentre presque exclusivement sur les classes des chevaliers et des druides, et surtout sur ces derniers 34 . Nous savons seulement que la position des classes subordonnées était nettement inférieure, aussi bien dans la dimension politique qu'économique et sociale (VI, 13, 1-2).

Or, si nous nous reportons à la situation hispanique, en premier lieu, l'existence de guerriers équivalents aux equites est indubitable, mais nous n'avons aucun témoignage sur leur pouvoir économique relatif. En deuxième lieu, je pense qu'il est probable qu'il y ait eu quelque chose de semblable aux druides, mais avec aussi quelques éléments spécifiques 35 , et nous n'avons aucune information sur leur pouvoir dans la société. Si nous ne pouvons pas assurer le parallélisme où il devrait le plus vraisemblablement se produire : dans les classes élevées (surtout à cause de l'orientation de nos sources), où devons-nous situer la plèbe hispanique par rapport à la plèbe gauloise ? Il faut y ajouter une différence temporelle.

On sait que la situation de dissensions et de déséquilibres internes dans laquelle se trouvait la société gauloise a facilité la conquête romaine. Mais nous devons nous demander dans quelle mesure ces déséquilibres n'étaient pas le résultat d'un type d'évolution qui n'avait pas encore eu lieu dans la protohistoire hispanique. En revanche, les déséquilibres propres à l'Hispanie ne seraient-ils pas une des conséquences d'une conquête prolongée dans le temps plutôt que le résultat d'une évolution autonome ? Si la réponse est affirmative, et il a dû y avoir un peu de cela bien que nous en ignorions le détail, nous ne pouvons pas accepter la comparaison avec la Gaule. Non seulement elle ne s'ajuste pas en ce qui concerne les classes supérieures, mais les deux situations dans leur ensemble paraissent très différentes (du moins en ce qui se réfère à leur

33. Voir Colectivismo Agrario en España, Madrid 1915 (1 ère éd. 1893), p. 423-4, et Tutela de Pueblos en la Historia, Madrid, [1895] p. 15.

34. Voir l'analyse détaillée de F. LE ROUX, Notes d'histoire des religions XX, Ogam 22-25, 1970-1973, p. 209-214.

35. Voir M.V. GARCIA QUINTELA, El Sacrificio humano. Adivinatorio céltico y la religión de los Lusitanos, Polis 3, 1991 ; F. MARCO SIMON, La Religión de los Celtiberos, in: I Symposion sobre los Celtiberos, Saragosse 1987, p. 54-74, p. 69-70 et G. SOPEÑA GENZOR, Dioses, Etica y Ritos, Saragosse 1987, p. 63-4. 
situation de plus ou moins haut degré de consensus ou d'équilibre social).

Un autre témoignage allégué en faveur de l'existence d'inégalités sociales dans la zone indoeuropéenne de la Péninsule se réfère aux Vaccéens, habitants de la partie occidentale du bassin du Douro. J. Caro Baroja le mentionne pour réfuter l'interprétation que J. Costa fait de Diodore $(V, 34,3)$, il la juge "politique" dans un sens "communiste" ou "socialiste" 36. Il s'agit d'un passage de Plutarque (Mul.vir. 248e-249b) où celui-ci narre le stratagème employé par les femmes des Vaccéens, habitants de Salamanque, pour empêcher que les clauses de la reddition soient appliquées et pour attaquer par derrière le vainqueur confiant, Hannibal. Il y est dit qu'à un moment donné les habitants de la cité y abandonnent armes, richesses et esclaves. Caro en déduit que le partage annuel des terres dont parle Diodore faisait seulement référence aux grands propriétaires vaccéens 37 .

Or, nous avons donc deux textes de nature différente, qui ne se complètent ni ne se recoupent en aucune manière. Diodore, pour sa part, constitue un rapport ethnographique dans lequel il recueille ce qui est le plus significatif de chacun des peuples qu'il mentionne. Il s'agit, en plus, d'un passage où l'ethnocentrisme n'est pas exagéré : les Celtibères sont hospitaliers et jouissent de ressources alimentaires abondantes, les Lusitaniens sont les plus vaillants des Ibères et les Vaccéens sont les chariéstaton des zones limitrophes 38 ; alors que, pour sa part, Plutarque reprend des exemples qui nourrissent sa thèse sur l'unité de l'aretè et, par conséquent, la possibilité de la trouver chez les femmes (Mul.vir. 242e-243e). Sa présentation des faits ibériques équivaut à celle qu'il fait de n'importe quelle cité, de n'importe quelle zone géographique, de n'importe quelle époque : il ne se soucie pas du type de source ou de la réalité à laquelle ils répondent. Malheureusement et malgré la

36. Pueblos, p. 318-9. Et voir mes "Peuples indigènes", p. 198-199 pour le sens de cette critique.

37. Pueblos, p. 319-20. récemment A.M. GONZALEZ-COBOS DAVILA, Los Vacceos. Estudio sobre los Pobladores del Valle Medio del Duero durante la Penetración Romana, Salamanque 1989, p. 38-40, suit Caro et ne mentionne pas Costa malgré les énoncés marxistes que soutient le livre.

38. Voir la première partie de mon "Viriathe" pour le reflet d'une idéologie trifonctionnelle indoeuropéenne dans ce témoignage. 
tentative de Caro, le texte de Diodore sur les Vaccéens ne peut être interprété que par lui-même ${ }^{39}$.

Notre troisième témoignage provient aussi de Diodore et depuis que A. Garcia Bellido l'a mentionné 40 , il est inévitablement repris comme preuve de déséquilibres sociaux. Il s'agit du passage où sont relatées les noces de Viriathe (XXXIII, 7, 1-4); la richesse d'Astoplas, représentant de la fiancée, est fortement soulignée et contraste avec le dédain que montre Viriathe pour les choses matérielles. D'autre part les humbles origines de Viriathe sont un lieu commun dans l'Antiquité 41. Peut-on légitimement déduire de ce texte l'existence de déséquilibres sociaux parmi les Lusitaniens ? Nous pensons que non, aussi bien pour des éléments internes du texte que pour l'existence probable d'un autre type de réalités institutionnelles.

En ce qui concerne le premier point : si nous acceptons pour un instant la perspective utilitariste régnante, il est tout simplement invraisemblable qu'un riche propriétaire comme Astoplas ait un quelconque intérêt à s'allier avec quelqu'un de grossier et d'origine humble comme Viriathe. Si, d'autre part, et comme le dénonce Viriathe lui-même, Astoplas avait de bonnes relations avec les Romains, quel genre de bénéfices rechercheraient les deux parties dans cette alliance ? Aucun des deux ne semble abandonner ses positions : Viriathe part à la montagne après la cérémonie et Astoplas conserve ses richesses intactes. Seule la femme se déplace et elle ne dit rien. L'aspect moralisant du texte est très clair et il est possible que Poséidonios, la source probable de Diodore, l'ait écrit avec cette intention 42 , de telle sorte que la précision d'autres détails

39. Dans l'étude de ce texte on apprécie aussi un aspect de la méthode de Caro : il ne hiérarchise pas les divers éléments qui constituent ce qu'il nomme "domaine culturel". Dans ce cas il doute que les Vaccéens puissent être celtes à cause du type de charrue employée traditionnellement dans la région occupée par ce peuple dans la protohistoire, sans considérer la langue qui pouvait y être parlée, voir Pueblos, p. 324-5.

40. Bandas y Guerrillas en las Luchas con Roma, Hispania V, $\mathrm{n}^{\circ} 21$, Madrid 1945, réédité dans le reading compilé par A. PRIETO ARCINIEGA, Conflictos y Estructuras Sociales en la Hispania Antigua, Madrid 1986, p. 13-60, voir p. 37-38.

41. Textes recueillis dans Fontes Hispaniae Antiquae, IV, p. 133-4.

42. Cf. J. MALITZ, Die Historien des Poseidonios, Zetemata Heft 79, Munich 1983, 123-4; plus amplement, L.A. GARCIA MORENO, Infancia, Juventud y Primeras Aventuras de Viriato, Caudillo Lusitano, 
serait superflue. Si le passage est moralisant, il ne peut pas servir de témoignage de l'état d'une société.

Ceci est mis en valeur si nous nous plaçons dans le plan institutionnel. Malheureusement, Diodore n'est pas assez précis en ce qui concerne le thème fondamental dans un mariage : les critères suivis pour établir l'alliance. Cependant une donnée se détache : Astoplas possède beaucoup de biens et cède une femme 43 . Pour sa part, Viriathe ne rejette pas seulement la richesse mais il dit qu'elle

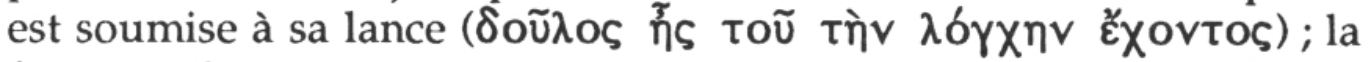
force, sa force, est au-dessus de la richesse, au-dessus d'Astoplas. Évidemment, si on pense que la hiérarchie sociale peut s'exprimer uniquement en termes d'usufruit de biens matériels et de contrôle de relations de production, ceci ne semble que de la simple rhétorique. Mais si nous considérons d'autres possibilités, comme l'expression de la différence sociale en termes symboliques, il n'en est pas ainsi. Ainsi, du point de vue de l'idéologie trifonctionnelle indoeuropéenne, telle que G. Dumézil l'enseigne, un guerrier se situera toujours au-dessus d'un représentant de la Troisième Fonction, malgré tous les biens que celui-ci rassemble, c'est ce que le texte fait dire très clairement à Viriathe.

Néanmoins, pour que cette interprétation soit possible l'existence d'un troisième élément qui représente la Première Fonction est indispensable. Le texte conservé de Diodore n'offre pas un personnage avec ces caractéristiques 44 . Mais cette idéologie trifonctionnelle s'apprécie dans la clôture de la cérémonie avec un

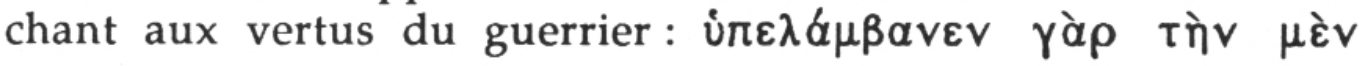

in: G. PEREIRA MENAUT (ed.), Acta $1^{\text {er }}$ Congreso Peninsular de Historia Antigua, Vol., II, Saint Jacques de Compostelle 1988, p. 373-382. Sur Poséidonios comme auteur de ces textes cf. W. THEILER (Hers.), Poseidonios, Die Fragmente, Berlin-New York 1982 , fr. $105 \mathrm{a}$ et $105 \mathrm{~b}$; MALITZ, Historien, p. 121-5. Pour leur part F. JACOBY, L. EDELSTEIN et I.G. KIDD, Posidonius, The Fragments, I, Cambridge $1989^{2}$, n'éditent pas le texte, Edelstein et Kidd en donnent les raisons p. XVII-XX. Voir aussi l'épisode étudié par BERMEJO BARRERA, La Calvicie de la Luna : Diodoro Siculo XXXIII, 7, 5-6 y la Posible Existencia de un Nuevo Mito Turdetano, in: Mitología y Mitos II, p. 45-56.

43. Qui, à l'encontre ce que l'on suppose, n'est pas forcément sa fille et peut bien être sa sœur, voir GARCIA QUINTELA, Viriathe.

44. Bien que Viriathe agisse sur les plans des Fonctions I et II, voir idem. 


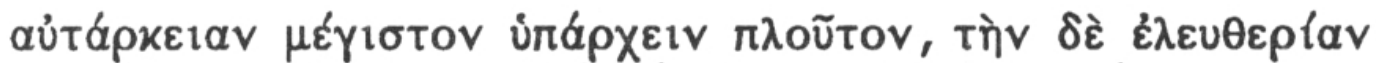

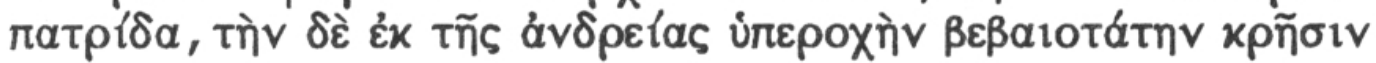
(XXXIII, 7, 3). Les idées reprises dans ce texte apparaissent formulées autrement dans un passage de Dion Cassius qui confirme ainsi au moins la force des idées dont dépendent les deux témoignages. En effet, Dion Cassius dit que Viriathe combattit oǔtع

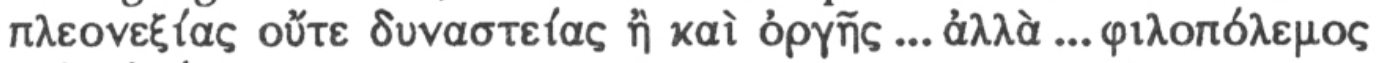

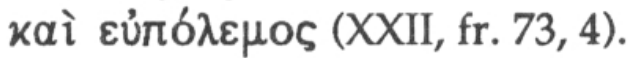

L'ordre conceptuel est le même dans les deux cas. Les deux premières vertus insistent sur l'autonomie du guerrier par rapport à la Troisième Fonction, reflétée par la mention de la richesse matérielle (ploûtos, pleonexía), et à la Première avec l'allusion à la souveraineté évoquée par la mention de la patrie ou d'une dynastie royale. Le guerrier se caractérise donc par la autárkeia, qui le rend indépendant des servitudes matérielles, et par la eleuthéria, qui empêche sa subordination à tout type de souveraineté définie en termes politiques ou religieux. Enfin, Diodore formule positivement la vertu la plus authentique du guerrier, celle qui définit sa prééminence sociale et son excellence, avec un terme appartenant au domaine de la Deuxième Fonction: la andreía 45. Pour sa part, Cassius, en suivant une formulation négative, indique un péché éventuel du guerrier, sa colère : orgès; puis, il insiste sur la pure vocation guerrière de Viriathe.

Les fonctions sont donc présentées selon l'ordre III-I-II, mais cette altération du canon hiérarchique est facilement expliquée par le contexte qui souligne la différence entre un guerrier et un possesseur dans le texte de Diodore et il se peut que Cassius ait lu quelque chose de semblable. De plus, ce dernier formule le thème profondément indoeuropéen des trois péchés (évités) du guerrier ; Diodore, quant à lui, l'explique de manière positive. D'autre part, lorsque celui-ci ne mentionne pas avec netteté une notion de souveraineté collective territoriale ou politique, il souligne le desideratum guerrier d'autonomie par rapport à la Première Fonction 46. Quoi qu'il en soit, l'action de Viriathe dans un domaine de souveraineté, et même d'exercice de la royauté, au sein de son ethnie est soulignée dans d'autres passages 47 .

45. Cf. D.S. XXXIII, 1,3 et $5 ; 21$.

46. G. DUMEZIL, Heur et malheur du guerrier, Paris $1985^{2}$, p. 72-79.

47. D.S. XXXIII, 1,$5 ; 21 \mathrm{a} ;$ XXXIV/XXXV, 4, 2 ; Flor. I, 33, 15. Etudiés en détail dans GARCIA QUINTELA, Viriathe, quatrième partie. 
Je ne pense pas que cette interprétation contredise celles avancées par d'autres auteurs d'après lesquels il faut voir dans Viriathe un représentant du "bon sauvage" selon des paradigmes idéologiques hellénistiques 48 . Dans ce moule intellectuel grec et philosophique peuvent se fondre des récits et des croyances d'origine indubitablement lusitanienne ${ }^{49}$. En tout cas, la richesse proverbiale d'Astoplas ne semble pas du tout représentative de déséquilibres hypothétiques dans les partages des biens, tout au contraire : elle reflète la subordination du ploûtos au kratos, en accord avec un état de civilisation dans lequel la morale et l'idéologie guerrières prédominent 50 .

Il existe un autre témoignage qui est parfois évoqué en faveur de la communis opinio 51. Il s'agit du récit que Plutarque fait des mesures prises par César pendant sa prêture en Hispanie Ultérieure en l'an 61-60 (Caes. 12). Elles furent guerrières (Plutarque se borne à les énumérer), et pacifiques : "il rétablit la concorde dans les cités et spécialement en assainissant les relations entre débiteurs et créanciers. Il ordonna que chaque créancier prît du débiteur annuellement les deux tiers de ses rentes et que le dernier tiers restât pour le propriétaire, jusqu'à ce qu'il eût remboursé la dette". Dans cette différence entre débiteurs et créanciers on a voulu voir une preuve de l'existence d'inégalités sociales en Hispanie. César serait l'un des Romains qualifiés de "bons", sensibles à la misère des autochtones.

Or, si nous suivons l'ordre de l'exposition de Plutarque, ces mesures ont un sens seulement si on les considère comme ayant été appliquées à la partie déjà conquise et romanisée de la Péninsule (le texte peut même reprendre en partie un édit de propréture et être

48. Voir les travaux de MALITZ et GARCIA MORENO cités supra n. 42.

49. Ceci est démontré par BERMEJO BARRERA dans Mitología y Mitos II, passim et idem, Mitología y Mitos de la Hispania Prerromana, Madrid 1982, passim.

50. J'étudie plus en détail toutes les questions en rapport au chef lusitanien dans mon "Viriathe".

51. Voir P. BOSCH GIMPERA et P. AGUADO BLEYE, La Conquista de Hispania por Roma, 218 a 19 a. de J.C. in: Historia de España Menéndez Pidal, tome II : España Romana, 218 a. de J.C.-44 de J.C., Madrid 1935, p. 3-286, voir p. 245-6 ; J.M. BLAZQUEZ, Ponencia sobre pueblos Prerromanos in: M. TARRADELL (éd.), Actas de la I Reunión de la Economía Antigua de la Peninsula Ibérica, Valencia 1969, p. 113-116, p. 114. 
donc uniquement applicable aux citoyens romains 52). Il serait certainement invraisemblable qu'il y ait là une sorte d'égalité sociale, après plus d'un siècle de domination romaine. En plus, ces mesures apaisent des maux, tel que le dit Plutarque, si elles ont un sens uniquement pour les couches les plus élevées et romanisées de la population. Rappelons simplement que, selon Tyrtée, les Messéniens soumis à Sparte cédaient la moitié du produit de leurs récoltes (fr. 5D) : le résultat de cette exaction peut être considéré comme l'on veut, mais jamais comme l'obtention d'une sorte de paix sociale. Si la mesure de César était applicable à d'amples couches de la société, il faudrait la considérer comme créatrice de différence sociale, à l'origine d'une espèce d'accumulation primitive de capital, à la source de relations de dépendance renforcées et, à moyen terme, de révoltes inévitables. C'est-à-dire tout à fait le contraire de ce que semble nous présenter Plutarque.

Si je ne me trompe pas, ce sont là toutes les sources alléguées en faveur de l'existence de forts déséquilibres sociaux. Leur examen nous empêche de les considérer de la manière traditionnelle. Cela ne signifie pas que, pour ma part, je pense que l'Hispanie indoeuropéenne protohistorique était un monde égalitaire, une sorte de paradis communiste primitif. J'essaie simplement d'affirmer que les sources employées ne sont pas correctes et que les méthodes d'analyse adoptées ne sont pas appropriées. En effet, la différenciation sociale et politique peut s'exprimer sous des formes très variées, comme le montre notre analyse du cas ViriatheAstoplas. Prendre seulement en compte le modèle interprétatif matérialiste ou utilitariste comme élément de définition de la hiérarchie sociale est, tout simplement, une preuve d'eurocentrisme colonialiste.

Au contraire, il se peut qu'il ait existé d'autres causes de déséquilibres sociaux, exprimés en termes matériels, qu'il faudrait étudier. Je me réfère, par exemple, au fait qu'une part importante des unités de production domestique d'une communauté donnée ont, inévitablement, tendance à dépendre des autres, tel que le montre la dialectique de la règle de Chayanov 53 . Normalement la culture, le terme étant employé dans un sens anthropologique, sert à établir des

52. Je dois cette idée à J.J. Segui Marco.

53. Voir A.V. CHAYANOV, La Organización de la Unidad Económica Campesina, Buenos Aires 1974 (Moscou 1925); et SAHLINS, Economía, p. 103s. et passim pour l'intégration des résultats de Chayanov dans la théorie anthropologique moderne. 
mécanismes de correction. Mais que se passerait-il si des territoires étaient soumis à une forte pression de la guerre et à des destructions presque incessantes?

De toute façon, il est possible que des situations comme celle que nous suggérons, bien que vraisemblables, ne puissent jamais être corroborées par l'analyse documentaire. En effet, si nos témoignages sont minutieux en ce qui concerne la séquence de la temporalité romaine, marquée par la substitution annuelle des magistrats et par les territoires où ils agissent, la situation est bien différente pour les peuples conquis. Ils sont toujours présentés comme s'ils sortaient d'une nébuleuse atemporelle, comme s'ils étaient un écran immobile sur lequel les Romains entrelacent leurs actions presque librement, en suivant les règles de personnages de genre. Or, les systèmes indigènes de classes d'âge ou de parenté, les oscillations de la richesse des différentes unités d'exploitation familiales, la dialectique causeeffet entre le jeu des institutions autochtones et une réalité qui change brutalement, tout cela a un sens seulement dans une séquence temporelle dont nous postulons le fonctionnement, mais dont les contenus concrets sont définitivement perdus 54 .

\section{Le destin de Galba}

Dans l'analyse des sources sur lesquelles s'appuie l'historiographie régnante, nous nous sommes intéressés à celles qui ont une relation avec la situation des sociétés conquises ; nous avons aussi fait allusion, à diverses reprises, aux inévitables changements provoqués par les processus de conquête. Les thèses "officielles", comme c'était prévisible, essaient également de trouver des appuis en analysant les actions des divers magistrats romains qui ont été en fonction dans la Péninsule. D'autre part, les sources à notre disposition semblent appropriées pour cette approche, puisqu'elles se situent pleinement dans des perspectives romaines.

Depuis Mommsen, on applique au phénomène de la conquête de la Péninsule une méthodologie positive qui a pour objectif l'établissement, de manière rigoureuse, des faits historiques (politico-militaires); puis, sur ces faits, on émet des jugements de valeur dûment pondérés 55 . Cette pratique historiographique

54. Le chapitre 3 du livre III de Strabon en offre un bon exemple : il y a un temps passé, l'an 137 av. J.-C. et un présent de pacification. Voir infra.

55. Voir C.V. LANGLOIS et C. SEIGNOBOS, Introducción a los Estudios Históricos, Buenos Aires 1972, p. 224-26 et 96-105. 
homologuée a été, et est, bien représentée dans notre cas. En suivant cette tendance, on a classé très tôt les actions des Romains et on les a considérées moralement négatives ou positives. En général il existe un accord sur la place de chaque personnage ou de chaque type d'actions. L'historiographie hispanique y introduit une nuance, elle se place exclusivement du point de vue des conquis (en réalité, des préjugés qu'elle a sur ceux-ci). On en arrive à la conclusion que sont "négatifs" les magistrats romains qui se sont distingués par leur violence, alors qu'au contraire on considère "positivement" ceux dont on dit qu'ils ont su saisir et ont essayé de résoudre les nécessités réelles des indigènes : leur situation de pauvreté. $\mathrm{Si}$, comme nous l'avons vu dans la première partie de cet article, cette prémisse se révèle fausse, une révision s'impose. Mais, auparavant, nous allons suivre les traces du modèle du méchant par excellence : S. Sulpicius Galba.

Ce personnage nous sert de modèle parce que, dans son cas, les règles fondamentales de la méthode positive régnante - prise en compte de toutes les sources et reconstruction des faits en accord avec le sens commun (qui n'est pas défini) - n'ont jamais été appliquées avec la rigueur nécessaire. Pour le démontrer nous étudierons les témoignages relatifs à ses actions dans la Péninsule et nous essaierons de reconstruire les faits auxquels il s'est trouvé mêlé, non pas selon notre sens commun, mais en accord avec les manières d'agir, grosso modo constantes, de beaucoup de Romains en Hispanie. Avançons la conclusion : les actions de notre homme ne se distinguent pas de celles de beaucoup d'autres qui sont considérés comme "bons".

En effet, le problème principal qu'a eu Galba (consul en l'an 144, après avoir été destiné en Hispanie Ultérieure en 151-150), si nous lisons les sources, a été son accroc avec la philosophie. Les faits négatifs qu'on lui impute, et qu'il ne laisse pas de reconnaître, sont les suivants : pendant l'exercice de ses fonctions en Hispanie, il fut responsable d'un grand massacre des Lusitaniens. Celui-ci, l'un des nombreux qui se produisirent pendant la conquête romaine du Bassin Méditerranéen 56 , eut un relief spécial seulement à cause du procès auquel Galba se trouva mêlé à son retour à Rome (en l'an 149) et à cause de la personnalité des opposants politiques qu'il dut affronter :

56. Voir W.V. HARRIS, Guerra e Imperialismo en la Roma Republicana, 327-70 a.C., Madrid 1989 (Oxford 1979), p. 51-2 et 262-3, ce à quoi il faudrait ajouter les témoignages sur l'Hispanie, recueillis à sa manière par GARCIA BELLIDO, Bandas y Guerrillas, passim. 
en particulier Caton 57 . Il faut y ajouter le fait que, tout au long de l'Antiquité, Galba eut toujours la réputation d'être un grand orateur. Les duels oratoires qui durent avoir lieu pendant ce procès remplirent, a posteriori, les pages des traités romains de rhétorique.

Pour étudier ce cas, nous devons partir de Tite Live, qui offre une information précise sur les faits, les actions légales et même les sources dont il disposait: le discours de Caton et trois autres de Galba lui-même (Per. 49). En ce sens, partir d'Appien, comme cela se fait normalement, n'a d'autre justification que la paresse du chercheur qui ne veut pas changer le livre qu'il a sur sa table. De plus, Tite Live nous permet de comprendre que Galba n'a jamais nié les faits qui lui étaient imputés. Deux de ses discours furent prononcés contre L. Escribonius, tribun de la plèbe, qui avait tout d'abord proposé une loi pour ramener de Gaule les Lusitaniens réduits en esclavage et vendus par Galba; (L. Scribonius tribunus plebis rogationem promulgasset, ut Lussitani, qui in fidem populo romano dedit ab. Servio Galba in Galliam venissent in libertatem restituerentur); dans le troisième, il justifie le massacre des Lusitaniens par la crainte qu'il avait de subir une attaque de leur part 58 . Il se limite, donc, à discuter l'interprétation qui est donnée de ses actions et l'opportunité des mesures de restitution proposées. Par conséquent, on déduit du texte de Tite Live qu'il y a eu deux groupes de Lusitaniens, les uns ont été réduits en esclavage et vendus et les autres ont été tués 59 .

57. La nature du délit commis, violation d'une dedito in fidem, reste au second plan, il semble plutôt un prétexte opportun sur lequel la tradition n'insiste pas. Voir A. HEUSS, Die völkerrechtlichen Grundlagen der römischen Aussenpolitik in republikanischer Zeit, Klio, Beiheft 31, Leipzig 1933, p. 60s. où il montre que, selon cette formule, le vaincu se rend sans réserves, bien qu'il ait le droit d'espérer un traitement correct. Cf. G. FREYBURGER, Fides, Etude sémantique et religieuse depuis les origines jusqu'd l'époque augustéenne, Paris 1986, p. 108-10 appuyant la thèse de Heuss et avec une autre bibliographie. Sur la perfidia de la part du dediticius voir idem, p. 143: la justification de Galba semble crédible, cf. M.V. GARCIA QUINTELA, El Sacrificio Lusitano. Estudio Comparativo, Latomus 51, 1991. Pour sa part R.C. KNAPP, Aspects of the Roman Experience in Iberia, 206-100 BC, Anejos de Hispania Antiqua IX, Vitoria, 1975, p. $44-5$ centre son analyse du cas Galba sur l'aspect juridique.

58. Aspect estudié dans GARCIA QUINTELA, "Sacrificio Lusitano".

59. Comme dans Val.-Max. IX, 6, 2. Voir infra p. 82-83. 
Néanmoins, aussi bien le point de vue de Galba que la distinction entre les deux groupes de Lusitaniens, sont complètement estompés dans la tradition relative à cet événement, tradition inspirée par Caton et hégémonique dans l'historiographie antique et moderne. On rappelle toujours que Caton participa à ce procès dans sa vieillesse lorsqu'il était imprégné de culture philosophique grecque 60 . De plus, l'auteur qui a repris le thème après lui fut P. Rutilius Rufus, l'un des premiers Romains à adopter le stoïcisme et qui se souvenait personnellement de Galba 61 . Cicéron recueille sa discussion du cas Galba en présentant Rufus comme "sage disciple des philosophes". Cependant nous lisons seulement des considérations à propos des bons usages devant les tribunaux : il se borne à reprocher à Galba d'avoir cherché à susciter le pathétisme et la pitié en se présentant devant le tribunal avec ses enfants et il considère qu'il a été acquitté grâce à cette feinte 62 .

Cicéron s'occupe à nouveau du cas Galba, cette fois plus amplement, dans son Brutus. Mais il établit uniquement des considérations de type rhétorique, le fond des faits ou leur appréciation morale sont sans importance 63. Néanmoins, l'évaluation que Cicéron fait du style de Galba a un certain intérêt pour nous : les discours conservés ne montrent pas la qualité oratoire de celui-ci (91). C'est dû au fait que certains "se croient plus capables de parler bien que d'écrire bien. C'est ce qui arrive très souvent à des hommes très doués mais dont l'éducation littéraire est insuffisante, comme précisément Galba" 64. Cicéron conclut son analyse en formulant une hypothèse sur l'éloquence naturelle de Galba qui aurait disparu dans la rédaction de ses discours (93-94).

60. Cic. De sen., II, 38 ; cf. G. DUMEZIL, La religion romaine archaique, Paris $1974^{2}$, p. 491-3.

61. Sur ce personnage, disciple de Panetius, juriste, historien et aussi connaisseur des choses de Hispania, où il combattit contre Numance aux côtés de Scipion, voir F. MÜNZER, R.E. Pauly-Wisowa, I, A, 1, 1914, s.v. Rutilius Rufus, $n^{\circ} 34$, cols. 1269-1280.

62. Reproche présent depuis Caton dans ses Origines, cf. Cic., d.or., I, 227-8.

63. Il souligne la force spéciale de l'art oratoire de Galba, il mentionne son délit (il tue les Lusitaniens fides interposita, 89) et il met en valeur le recourś à la pitié des Romains ainsi que le point de vue de Caton sur cela (90).

64. 92: Alios, quod melius putent dicere se posse quam scribere, quod peringeniosis hominibus neque satis doctis plerumque contingit, ut ipsi Galbae. 
Si l'on ne prend pas en compte cette analyse de Cicéron, fortement teintée de logocentrisme, mais qui tente aussi une approche sérieuse de Galba, il est impossible de comprendre les témoignages postérieurs. On y trouve seulement les effets d'une rhétorique de souche philosophique, dont l'origine se situe chez Caton lui-même, unis à une exposition incomplète ou contradictoire des faits imputés à Galba (en parlant toujours de Tite Live). A ce modèle se plient les considérations rhétoriques de Quintilien (ins. or., II, 15, 8) ou le récit "historique" d'Orose (IV, 21, 3 et 10).

D'autres sources essaient d'ajouter un récit détaillé des événements, sans correspondre ni à la tradition oratoire romaine teintée de philosophie grecque (Caton, Rufus, Cicéron), ni à la pure et positive akribeia historique développée par Tite Live. Elles mêlent les deux influences et ont pour résultat l'histoire moralisante que nous lisons encore actuellement. Mais ceci se fait au prix d'une reconstruction plausible des faits. Voyons comment ceux-ci se présentent.

Pour Suétone, Galba provoque la guerre de Viriathe quand il fait massacrer traîtreusement 30.000 Lusitaniens 65. Chez Appien, nous lisons un exposé plus détaillé des faits. Premièrement, il signale la tromperie de Galba en lui faisant prononcer un discours dans lequel il promet des terres divisées en trois lots 66 . Les Lusitaniens le croient et vont le trouver, il les partage en trois groupes en donnant à chacun d'eux une vallée. Les ayant ainsi répartis, il les massacre les uns après les autres; seuls quelques uns, dont Viriathe, s'échappent 67. Enfin, Valerius Maximus dit que Galba convoqua les habitants de trois cités lusitaniennes, il prit 8.000 jeunes qu'il désarma et il en disposa en tuant les uns et en vendant les autres 68 .

Pour ne pas entrer dans des reconstructions des faits plus ou moins ingénieuses ou dans un examen des sources, toujours hasardeux vu le type de documents disponibles, voyons ce que fit Caton contre les habitants de Vergium pendant son consulat en l'an 195. Tite Live raconte que certains d'entre eux demandèrent de l'aide afin de libérer leur cité qui était aux mains des voleurs. Devenu maître des lieux, Caton divise la population en trois groupes : ceux qui ont combattu à

65. Galba, III : triginta Lusitanorum milibus perfidia trucidatis.

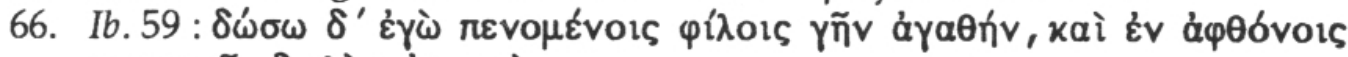

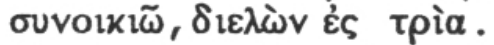

67. $I b ., 60$. Dans $I b ., 61$ Appien rappelle que l'armée de Viriathe était composée de survivants des actions de Galba et Lucullus.

68. IX, 6, 2. Cf. Val.-Max. VI, 4, 2 et VIII, 1, $2 ; 7,1$. 
son côté sont libres et il leur restitue leurs biens (liberos esse cum cognatis suaque habere iussit); il ordonne que les autres habitants soient vendus par le questeur (Vergestanos ceteros quaestori ut venderet imperavit); et envoie les bandits au supplice (praedonibus supplicium sumpsit) 69 . D'autre part, rappelons que, selon Cicéron (Caecil. 66), après son consulat Caton fut considéré par les Espagnols comme leur protecteur à Rome, en accord avec la nomination sénatoriale.

Or, cette tripartition des vaincus rappelle particulièrement les actions de Galba. Tite Live et Valerius Maximus coïncident sur l'existence de deux groupes exactement superposables à deux groupes d'habitants de Vergium : ceux qui sont réduits en esclavage et ceux qui sont tués. Le troisième est plus flou mais il n'en est pas moins présent : Valère-Maxime rappelle que Galba s'occupe uniquement d'une classe d'âge, la juventus des trois cités, c'est-à-dire que les restants seraient libres; pour sa part Appien rappelle que certains ont pu s'échapper, parmi lesquels Viriathe. Avec ceci seulement, le parallélisme entre les mesures de pacification de Caton et Galba est déjà très grand; mais peut-on franchir un autre pas et considérer les Lusitaniens épargnés comme des "collaborateurs" ? Nous ne pouvons pas répondre avec sûreté et ce n'est pas fondamental pour notre argumentation. Malgré tout, il convient de rappeler les vélléités philoromaines d'Astoplas, le parent par alliance de Viriathe (D.S. XXXIII, 7, 4). Que celui qui devient par la suite chef lusitanien ait échappé de la boucherie grâce à ses relations est une simple hypothèse, mais elle n'en est pas moins viable dans une situation de frontière instable, comme celles évoquées par S.L. Dyson 70 .

69. Liv. XXXIV, 21. Selon J. MARTINEZ GASQUEZ, La Campaña de Catón en Hispania, Barcelone 1974, p. 146-7, 170 et spécialement 69-70, la source de Tite Live serait Caton lui-même.

70. Spécialement dans : Native Revolts in the Roman Empire, Historia 20, 1971, p. 239-274, passim, où il souligne que ceux qui dirigèrent les cinq révoltes étudiées avaient en commun un degré relativement élevé d'acculturation. Ainsi l'analyse antérieure n'est pas contredite (supra p. 73-76). Là il s'agissait de délimiter l'idéologie guerrière qui soutenait l'action de Viriathe, ici il s'agit d'une hypothèse sur ses relations familiales réelles. 
Ainsi, Galba divisa un groupe nombreux de Lusitaniens en trois 71 et il se borna à reproduire l'action que son ennemi le plus célèbre avait exécutée quelques années auparavant 72 . Si l'un est taxé de "méchant" et l'autre de "bon", cela n'a rien à voir avec les faits auxquels ils ont été mêlés car ils sont presque identiques. De plus, même lorsqu'il eut lieu, le procès de Galba n'a pas dû avoir une importance majeure, comme le prouve son élection comme consul en 144 73. Ce qui change de l'un à l'autre c'est leur fortune historiographique respective. Du point de vue de l'analyse des bases documentaires des thèses historiographiques en vigueur, c'est précisément la distinction entre les faits auxquels a été mêlé Galba et sa fortune pour la postérité que la méthodologie positive aurait dû faire depuis longtemps. Comme il n'en fut pas ainsi, les préjugés philosophiques de quelques Romains cultivés se sont convertis en instruments analytiques pour la compréhension de la conquête d'Hispanie.

Mais cette appréciation n'est pas seulement le résultat du parallélisme entre les actions de Galba et de Caton. C'est simplement le cas le plus précis et spécialement significatif, puisque tous deux se sont opposés directement. D'autres généraux romains ont vécu des faits semblables et ont établi des mesures équivalentes. Ainsi, T. Sempronius Gracchus (l'un des "bons" de l'historiographie), dans une action contre Complega en 179, tue la plupart de ses habitants et constitue une nouvelle entité politique avec les survivants (App. Ib., 43) : il manque seulement le groupe de ceux réduits en esclavage du modèle Caton-Galba. Pour sa part, Sertorius (un autre Romain "compréhensif" avec les indigènes), dans ses

71. Ceci est rappelé clairement chez Suétone : 30.000 morts; Appien : 3 lots de terre et trois groupes; Valère-Maxime: proviennent de trois cités.

72. G. DE SANCTIS, Storia dei Romani, vol., IV, $2^{\text {ème }}$, Florence 1969, (Turin 1923), p. 466-469, évalue la politique de Caton envers Carthage comme très semblable à celle de Galba envers les Lusitaniens: "Galba, écrit De Sanctis (p. 469), pourrait avoir répondu à Caton en se déclarant son disciple" ; cf. H.H. SCHULARD, Roman Politics, 220-150 B.C., Oxford $1973^{2}$, p. 235-6 et 271.

73. Appien lui-même, $I b ., 55$, un des auteurs les plus durs avec Galba, rappelle que Lucullus, malgré ses fautes n'a jamais été jugé. Cf. HARRIS, Guerra e Imperialismo, p. 272-3 et SCHULARD, Roman Politics, p. 194-198, sur M. Popilius Lénas et les Statelli, un cas très semblable et sans conséquences graves non plus. 
quartiers d'hiver à Castulum, punit une attaque des habitants du lieu en tuant les adultes et en réduisant les autres en esclavage (Plut., Sert., 3) : dans ce cas c'est le groupe des survivants qui manque.

Les mesures imposées par le jadis "modéré" César après sa victoire sur les partisans de Pompée en Hispanie (en 45) répondent aux mêmes normes, bien qu'il n'agisse plus sur des indigènes barbares mais sur des provinciaux en processus avancé de romanisation. A Séville et Cordoue, il tue les résistants et vend les autres, à Munda et dans d'autres cités il tue beaucoup de monde, il pille tous les habitants, il dépouille certains de leurs terres et lève des impôts ; il concède à ses partisans des droits juridiques et des terres (D.C. XLIII, $39,2-5)$. Nous retrouvons, sous une forme plus détaillée, le même schéma que chez le tandem Caton-Galba: morts, réduits en esclavage, survivants/favorisés.

Si les mesures que prend Auguste contre les Cantabres changent un peu, c'est dû simplement au fait qu'elles s'adaptent à la situation et qu'il ne s'agit pas d'un territoire tentant. De toute façon, en plus des combattants morts pendant la guerre, Auguste vend des prisonniers, garde des otages et agit sur le territoire en obligeant les indigènes à établir des habitats dans la plaine 74 .

En définitive, tous les Romains qui entreprirent des mesures de répression et de contrôle territorial ont agi, malgré quelques variantes, en suivant des normes de comportement stables tout au long de la période de conquête. L'action de César sur des populations soumises à Rome depuis longtemps empêche de considérer certaines mesures, par exemple la redistribution des terres, comme la conséquence d'une compréhension généreuse des problèmes économiques des vaincus 75 . Il s'agit toujours d'établir des politiques de domination efficaces sur des peuples et des espaces : il n'y a pas de Romains pires ou meilleurs, comme le veut l'historiographie régnante, il y a des conquérants qui essaient de s'assurer, avec plus ou moins de succès, la soumission des vaincus. Pour finir de démontrer ceci, nous devons affronter la dernière ligne de résistance des thèses

74. Flor. II, 33, 52 et 59. Cf. D.C. XXXVII, 52-3: César à la recherche d'un casus belli ordonne que les Lusitaniens descendent dans la vallée, et D.C. LIV, 11, 1: Agrippa oblige les Cantabres à s'installer dans les vallées.

75. Cf., s'il y a quelque doute, les terres réparties par César aux indigènes en Gaule seulement après qu'il ait obtenu des victoires sanglantes, B.G., I, 28 ; III, 16. 
"officielles" : la politique de fondations urbaines comme mesure contre la misère des indigènes.

\section{La fondation de cités}

Le fait que les magistrats romains en fonction dans la Péninsule pendant l'époque républicaine appliquent, avant la lettre, une sorte de "doctrine sociale de l'Église" semble, à première vue, surprenant. En effet, il paraît étonnant qu'alors que généralement ils recherchent la gloire de la cérémonie du triomphe 76 et qu'ils sont avides des biens des conquis 77 , certains d'entre eux, même si ce ne sont que quelques uns, aient échappé à ces manières d'agir propres à leur ethnos national et de classe. Mais, comme de telles choses peuvent être lues fréquemment dans l'historiographie en vigueur, il convient, une fois de plus, d'examiner les sources sur lesquelles ces interprétations se fondent pour les évaluer.

Nous pouvons partir du texte du Bronze de Lascuta (= CIL II 5041), célèbre "pour sa valeur pour l'histoire politique et sociale de l'Espagne Antique" selon Bosch Gimpera et Aguado Bleye 78. Il s'agit d'une intervention de Paul Emile sur les indigènes soumis en l'an 189. Voici le texte.

L. Aimilius L. F(ilius) impeirator decreivit ut ei quei Hastensium servei in turri Lascutana habitarent, leiberei essent, agrum oppidumqu(e), quod ea tempestate posedisent, item possidere habereque iousit, dum poplus senatusque Romanus vellet.

76. Voir HARRIS, Guerra e Imperialismo, p. 10-40 sur l'ethos de l'aristocratie romaine. Sur le symbolisme royal et divin qui entourait le triomphateur voir H.S. VERSNEL, Triumphus. An Inquiry into the Origin, Development and Meaning of the Roman Triumph, Leiden 1970, p. 56-92.

77. Voir en dernier lieu la récente thèse doctorale de J.J. FERRER MAESTRO, El Estado y la Participación Privada : los inicios del Régimen Provincial Romano en Hispania, Valencia 1987, sous presse, dans laquelle il réalise une analyse minutieuse du système de transfert de richesses de la Péninsule à Rome pendant toute la période de conquête.

78. Dans: Comienzos de la Resistencia de España al Yugo Romano in: Historia de España dirigida por R. Menédez Pidal, vol. II : España Romana 218 a de J.C. - 414 de J.C., Madrid 1935, p. 69. 
On en a déduit que les esclaves des habitants d'Hasta qui résidaient dans la tour de Lascuta, seraient libres sous la tutelle de Rome 79. Il s'agirait donc d'une intervention sur le tissu social d'une communauté. Mais ceci ne semble pas être une interprétation exacte : les esclaves présumés "possédaient" agrum oppidumqu(e) et on leur garantissait cette possessio, c'est-à-dire un habitat fortifié et les champs qui l'entouraient: la turri Lascuta. C'était l'ensemble de cette communauté qui, du point de vue du rédacteur de l'inscription, était en relation de dépendance avec Hasta. S'il en est ainsi, il s'agirait d'une intervention sur le cadre politique, qui aurait pour but d'appuyer d'éventuelles tendances centrifuges présentes dans des communautés politiques d'entité plus importante 80 .

Pensons un moment aux expériences complexes et mieux connues des poleis grecques. Le modèle en vigueur évoque une relation semblable à celle qui existe entre hilotes et Spartiates. Pour notre part, nous suggérerions le rapport existant entre Thèbes et les autres communautés de la confédération de Boétie, avec des tensions centrifuges et centripètes qui sont alternativement hégémoniques. Il pourrait, éventuellement, exister un modèle mixte représenté par le cas messénien : à la tension sociale s'ajoute la tension politique. C'est peut-être vrai, mais nous allons nous intéresser seulement à l'aspect politique.

79. Idem, p. 69 ; GARCIA BELLIDO, Bandas y Guerrillas, p. 38 n. 44 ; cf. H. GALSTERER, Untersuchungen zum römischen Städtewesen auf der Iberischen Halbinsel, Berlin 1971, p. 14-15.

80. Dans le volume collectif Epigrafía Hispánica de Epoca RomanoRepublicana, Saragosse 1983, Luis A. GARCIA MORENO, Sobre el Decreto de Paulo Emilio y la Turris Lascutana (CIL., 12, 614), p. 195-218 ; F. MARCO SIMON, La manumisio oficial de Emilio Paulo en el Marco de la Politica Internacional Romana del siglo II a.C., p. 219-225 ; A.M. CANTO, Un Nuevo Documento de Paulo Emilio en la Hispania Ulterior (CIL, I, $546=C I L$, II, 1119), p. 227-234 et F. MARTIN, La Formula Populus Senatusque Romanus en el Bronce de Lascuta, p. 235-238 réalisent diverses contributions qui renouvellent l'étude de ce document. Garcia Moreno reprend la bibliographie antérieure et critique les thèses en vigueur p. 201. Les appréciations qui suivent peuvent peut-être contribuer à cette nouvelle discussion sans prétendre annuler tout ce qui a été dit à ce propos. Voir aussi F. WULFF ALONSO La Fundacion de Carteya. Algunas Notas, Studia Historica 7, 1989, p. 43-57, p. 52-53 avec des affirmations qui convergent avec ce qui suit. 
L'évocation du monde grec est opportune si nous tenons compte du fait qu'un bon nombre des documents que nous étudions ont été rédigés selon les catégories qui émanent de la réflexion politique grecque. En effet, chez Strabon, nous lisons aussi bien un appui à l'interprétation donnée du Bronze de Lascuta que l'application d'une politique analogue. Il s'agit du récit de la campagne menée par D. Junius Brutus dans le Nord-Ouest de la Péninsule en l'an 137 81. Les

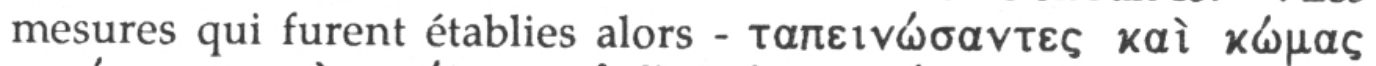

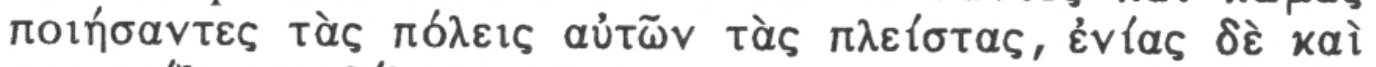

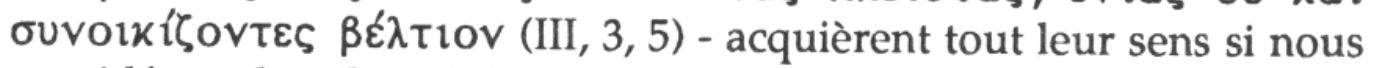
considérons la valeur politique précise de chacun des tours employés. Il s'agit d'humilier les vaincus en intervenant sur leurs structures politiques. Tout d'abord, on réduit les communautés politiques à leurs unités élémentaires : les bourgades. Le vocabulaire et le processus sont, d'une part, inverses à ceux théorisés par Aristote pour expliquer la constitution de la polis (Pol., 1252 b 15-35) et traduisent, d'autre part, en grec l'action de Paul Emile : Lascuta est une kóme de la pólis Hasta, qui est désagrégée après une action militaire. Mais, en plus, poursuit Strabon, on a réalisé des regroupements politiques (sunoíkizo): on essayait probablement de favoriser les positions d'éléments proromains 82.

En tout cas, l'objectif était d'obtenir la paix en modifiant le système politique pour l'affaiblir. Ceci se voit clairement si nous nous intéressons au destin des deux interventions romaines que nous venons de mentionner. D'une part, Lascuta est évoquée par Pline comme une simple cité stipendiaria du conventus de Cadix ( $\mathrm{NH}$. III, 15). D'autre part, les mesures de Brutus n'ont pas empêché que, après la campagne d'Auguste, la présence de deux légions soit nécessaire

81. Le chapitre 3 du livre III de Strabon correspond à des données provenant de l'époque de cette campagne, il mentionne Brutus dans les paragraphes 1, 2, 4, 7. La source est Poséidonios, cité dans les paragraphes 3 et 4 . Seul le paragraphe 8 est une description de l'époque de Strabon et dont il est l'auteur.

82. Voir infra les études des cas de Graccuris et d'une cité anonyme fondée par Marius à proximité de Colenda. D'autre part, serait-il convenable de penser que Paul-Emile, personnage très hellénisé affronte la situation de Lascuta en se guidant avec des catégories helléniques? 
dans ce territoire (Str. III, 3, $8 ; 4,20$ ), et que le processus d'urbanisation soit plus tardif 83 .

L'activité complexe de T. Sempronius Gracchus dans la Péninsule Ibérique (180-179), que l'on loue traditionnellement pour son caractère social, s'inscrit parfaitement dans le cadre que nous venons d'indiquer. D'une part, il mène une campagne militaire dont les nombreuses victoires lui ont valu le triomphe $84:$ il détruit beaucoup d'établissements indigènes, quelle que soit leur catégorie 85 . Appien $(I b ., 43)$ raconte en détail le cas de Complega, où, après avoir tué un bon nombre d'indigènes, Gracchus a fondé une

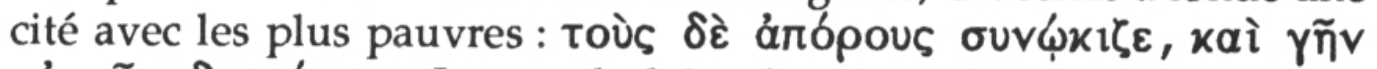

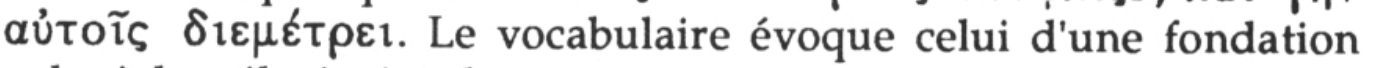
coloniale; il s'agit, dans ce cas, de remplacer les structures politiques détruites au cours de la campagne (il a tué la plupart des 20.000 combattants qui l'ont attaqué) par d'autres favorables aux Romains, le fait que les bénéficiaires soient désignés comme "pauvres" est seulement un effet de rhétorique 86.

Le récit de Tite Live (Per., 41) fait de la fondation de Graccuris est moins satisfaisant ; il se borne à dire que Gracchus a donné son nom à la fondation, (oppidum) monimentum operum suorum. Festus ajoute que dans ce lieu existait un habitat (urbs) indigène appelé Ilurcis (p. 77 M. = 86 L.). Si nous nous en tenons strictement aux sources, nous pouvons seulement parler du changement de dénomination d'une localité indigène qui a obtenu, très tard, un statut privilégié 87 .

83. Cf. A. TRANOY, La Galice romaine. Recherches sur le Nord-Ouest de la Péninsule Ibérique dans l'Antiquité, Paris 1981, p. 190-220.

84. Liv. Per., 41 ; App. Ib., 43.

85. Str. III, 4, 13, recueilie la polémique de Poséidonios contre Polybe. Celui-ci avait affirmé que Gracchus avait détruit 300 póleis qui, selon Poséidonios, seraient de simples púrgoi. Flor. I, 39, 9 résout la polémique à la manière de Salomon en parlant de urbes et en réduisant leur nombre à 150 ; Liv. XL, 49 mentionne 130 oppida dont il accepte la reddition.

86. Voir supra l'analyse de Diod., V, 34, 6 et n. 75 avec les actions de César en Gaule; et infra l'examen des cas de Contrebia et la fondation anonyme près de Colenda.

87. GALSTERER, Untersuchungen, p. 13 compare celle-ci avec la fondation de Pompaelo par Pompée plus tard (p. 14), il étudie aussi le cas de Iliturgis, p. 13, n. 53 de fondation attribuée à Gracchus par une inscription tardive. 
Néanmoins, le contenu des accords imposés aux vaincus est clair : on leur exige des tributs, des troupes auxiliaires et on leur interdit de construire des murailles 88 . Cette dernière condition a une influence sur la capacité que peuvent avoir les indigènes pour garder une indépendance politique réelle qui, entre autres choses, implique la capacité d'établir de nouvelles structures plus puissantes. Le casus belli qui débouchera sur la guerre de Numance se centre sur ce point : Segueda conduit des cités plus petites (brachutéras póleis) à s'établir en un même lieu et à s'entourer de murailles 89 . Une fois de plus ces brachutéras póleis nous rappellent Lascuta et les kómai de Strabon: on prétend toujours s'assurer l'affaiblissement des entités politiques indigènes 90 .

Les derniers sursauts des guerres contre Viriathe menés par G. Servilius Caepius (cos. 140) reçoivent un traitement très proche de celui infligé par Gracchus à Complega. Après avoir défait Tantale, successeur de Viriathe, il prit les armes des Lusitaniens et leur donna des terres pour qu'ils ne fussent pas obligés de voler 91 . Pour sa part, Diodore indique que Caepius céda aux Lusitaniens chóran kaì pólin (XXXIII, 1, 4). En tout cas, il n'y a pas plus de précision sur cet

88. Mais voir S. SZADECZKY-KARDOSS, Nouveau fragment de Polybe sur l'activité d'un proconsul romain, distributeur de terres en

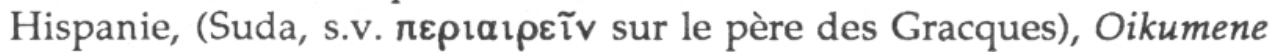
1, 1976, p. 99-107; selon l'auteur le texte de la Suda se réfère à Gracchus et témoigne de son intervention devant le Sénat en faveur des Celtibères. Le modèle général manié par l'auteur est celui de la communis opinio.

89. App. $I b ., 44$; D.S. XXXI, 39 ; cf. App., Ib., 100, Didius interdit la construction des murailles de Termesa.

90. Il est possible que l'action de Gracchus s'inspire du modèle d'Alexandre (mentionné par A. SCHULTEN, Fontes Hispaniae Antiquae, III, p. 223). Quoi qu'il en soit, ce modèle est plus clair dans le cas de Pompée, voir P. BRIANT, Brigandage, dissidence et conquête en Asie achéménide et hellénistique, DHA 2, 1976, p. 163-279, p. 204-5. Cf. Plut. Pomp., 28, 4-5 et Str., VIII, 7, 5. Cet exemple a pu également opérer dans la fondation de Pompaelo. La vigueur de ce modèle de conquête d'Alexandre consistant en l'établissement de certaines places fortes dans les territoires soumis est aussi possible, c'est clair dans le cas d'Olisipo (Lisbonne) fondée comme base militaire (Str., III, $3,1)$ mais, pourrait-il s'étendre à d'autres cités alliées comme Pompaleo, Complega, Graccuris, ou la fondation de Marius près de Colenda?

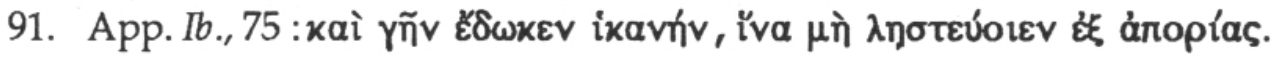


éventuel établissement urbain. Une fois de plus, les Romains consentent seulement à ce que leurs ennemis vaincus s'organisent en structures politiques mineures et subordonnées.

Si nous acceptons le texte d'Appien comme un reflet fidèle de la réalité, le cas d'une cité dont le nom n'est pas donné et qui se situe près de Colenda montre la faiblesse de l'interprétation traditionnelle, qui voit dans la fondation de cités et la concession de terres le résultat d'une simple politique sociale. En effet, l'auteur raconte que cette cité était habitée par des Celtibères de provenance variée (migódes Keltibéron), alliés de Marius dans ses campagnes contre les Lusitaniens et qui, comme récompense, avaient reçu cet établissement avec l'accord du Sénat. Donc, nous sommes face à une espèce de synécisme de groupes de populations proromains. Bien que cette fois les indigènes apparaissent sous un angle favorable, comme alliés de Rome, il est impossible de comprendre cette situation sans un processus préalable de destruction et de désagrégation des entités ethniques et politiques pendant la conquête. Quoi qu'il en soit, selon Appien, les habitants de cette communauté, bien qu'ils eussent, théoriquement, reçu les terres nécessaires pour subsister, se consacraient encore au "banditisme" à cause de leur pauvreté (Ib.,

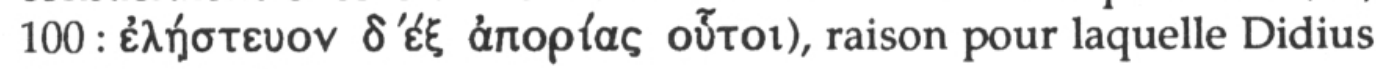
décida d'en finir avec eux.

La forme sous laquelle il le fait reproduit presque exactement le récit d'Appien à propos de Galba : Didius offre aux notables la répartition de la chóra de Colenda à cause de leur pauvreté ; ceux-ci acceptent et vont le voir ; alors, il les partage en mettant les hommes d'un côté et les femmes et les enfants de l'autre ; puis il les tue tous ; de retour à Rome, on l'honore d'un triomphe (App. Ib., 100). Or, rappelons que dans le cas de Galba, avec d'autres témoignages à notre disposition, nous considérions que la version d'Appien était imprécise et moralisante ${ }^{92}$. Comme à présent nous manquons d'autres références nous nous bornerons à suggérer que Didius a appliqué quelque chose de semblable à la répartition des vaincus en des groupes distincts selon le modèle Caton-Galba. Plus tard, Appien, ou une tradition intermédiaire, a exprimé cette situation en suivant le modèle narratif appliqué à Galba : avec le mélange que nous examinions d'intention moralisante unie à l'imprécision dans le registre des faits.

92. D'autre part, le triomphe concédé à Didius, selon Appien lui-même, met en valeur le caractère exceptionnel des circonstances qui ont entouré le procès contre Galba. 
Qu'est-ce qui a pu se passer pour que la communauté fondée par Marius, sûrement dans les meilleurs conditions puisqu'il s'agissait de ses alliés, se voie réduite à une misère telle que ses habitants en sont amenés à pratiquer le banditisme? Du point de vue de l'historiographie traditionnelle, ou bien l'on reconnaît que Marius et ses alliés sont bêtes (parce qu'ils établissent une communauté sans garanties de survie) ou bien la situation est tout simplement absurde : un groupe guerrier a reçu une concession de terres, mais il pratique le banditisme parce qu'il vit dans la pauvreté. Si nous nous dégageons de la considération exclusive de certaines conditions économiques mal articulées dans nos témoignages, le processus est, au contraire, intelligible : la communauté synoicisée par Marius a eu du succès, elle a réussi en tant qu'entité nouvelle au-dessus des éléments constitutifs dissemblables, elle a atteint une entité politique spécifique. Cette situation est perçue à travers le processus de différenciation et d'individualisation de la communauté dont l'expression était la guerre et l'ensemble des institutions qui l'entourent 93 .

Il ressort de cet examen que l'intervention romaine sur les habitats et les structures politiques indigènes répond à la nécessité de contrôle politique des vaincus. De plus, il est possible que, dans des cas concrets, on ait considéré les conditions sociales. Mais, devant l'insistance de la part de la communis opinio sur ce dernier point, il se révèle nécessaire de mettre en valeur le fait que les situations évoquées montrent que les fondations se produisent ou bien après une grande déroute des indigènes (Complega), ou bien sont le résultat de l'encouragement des tendances centrifuges (Lascuta, Nord-Ouest) ou

93. Les réformes de Clisthène à Athènes, intégrant des communautés avec des tendances centrifuges et une population nouvelle (Arist. Ath. pol., 21, 3-4; cf. P. LÉVÊQUE et P. VIDAL-NAQUET, Clisthène l'Athénien, Paris 1964, passim), manifestent tout de suite une efficacité militaire notable (Hdt. V, 77 ; cf. $\mathrm{H}$. VAN EFFENTERRE, Clisthène et les mesures de mobilisation, $R E G, 89,1976$, p. 1-17 insiste sur cet aspect). Si la comparaison avec Athènes peut sembler très élevée, d'autres peuples "exotiques" donnent des preuves d'une capacité politique pour établir des synécismes comparables, voir par exemple P. CLASTRES, Chronique des indiens Guayaki, Paris 1972, p. 36-41, 165-9 et passim; cf. P. CLASTRES, Investigaciones en Antropología Política, Barcelone 1981, (Paris 1980), p. 181-216, pour une analyse de la violence comme expression de l'identité politique. Si nous retournons en Grèce, ceci avait déjà été exprimé par Platon, Prot., 322 b, et Aristote, Pol., 1254 b 30-32, pour eux la guerre se situe dans la politique. 
de l'interdiction de réalisation des forces centripètes (Segueda). Il s'y ajoute le traitement de faveur reçu par les indigènes alliés (probablement Graccuris, fondation de Marius, Pompaelo), mais seulement s'ils acceptent leur soumission à Rome. Enfin, il faut prendre en considération les fondations de bases militaires qui ont eu pour résultat des communautés (Olisipo). Cette situation présente des analogies avec la fondation de colonies latines dans la zone méditerranéenne de la Péninsule. Bien que celles-ci jouissent d'un statut juridique privilégié, leurs fonctions sont semblables à celles des communautés indigènes : contrôle du territoire et récompense aux habitants pour les services rendus 94 , elles allègent en même temps les déséquilibres sociaux existant en Italie.

Pour finir, bien que ceci ne nous semble pas une explication satisfaisante, nous ne pouvons laisser de côté l'insistance des témoignages que nous lisons sur la pauvreté et la faim de terres des "bandits" de la Péninsule. Appien est l'un des défenseurs les plus fervents de la thèse du manque de terres comme cause de la "subversion" : les Lusitaniens se rebellent parce qu'ils manquent de terre et ceux de Complega en ont aussi besoin (Ib., 42); nous avons déjà vu comment Gracchus résout la situation de ces derniers (supra p. 89-90); en ce qui concerne le cas Galba, Appien est la seule source qui fait tourner l'affaire autour de la faim de terres (Ib., 59-60 cf. supra p. 80 et 82 ). Après Galba, Gaius Vetilius promet aussi des terres à Viriathe, ce que celui-ci refuse $(I b ., 61)$; F. Maximus Servilianus établit également un traité avec Viriathe selon lequel il garantit leurs terres aux partisans de celui-ci $(I b ., 69)$; nous avons déjà vu que la pacification de Tantale de la part de Caepius et le cas de la cité proche de Colenda renvoient aux mêmes motifs ( $I b$., 75 et 100).

Quel poids peut être donné à l'ensemble de ces témoignages ? Pour répondre à cette question il faut situer ceux-ci dans leur contexte. Appien est à la fois (a) notre source la plus complète sur la grande période de la conquête de la Péninsule; (b) l'une des plus distantes aux événements signalés et le produit d'une reconstruction purement livresque menée à bien à Alexandrie, bien qu'il ne faille pas dédaigner sa capacité intellectuelle et son originalité 95 ; (c) une des

94. Voir GALSTERER, Untersuchungen, p. 15-16 sur Cartela, Corduba, Ilerda, Palma et Pollentia ; cf. KNAPP, Aspects, p. 53-4 et 105-120.

95. Appien vécut entre 95 et 165 de notre ère. Sur son travail et ses sources, voir E. SCHWARTZ, R-E Pauly-Wisoa, II, 1, 1985, s.v. Appianus, cols. 216-237, contra H. WHITE, Appian's Roman History I, LCL, Londres-New York 1912, p. IX-X. F. WULFF ALONSO, Apiano: 
sources les plus désespérantes en ce qui concerne la Quellenforschung : il ne mentionne aucune autorité. Sans prétendre faire une recherche sur ses sources, il faut, pour comprendre le texte d'Appien, le placer dans la perspective que marquent les témoignages de l'époque augustéenne : Diodore, Strabon et Tite Live 96 . Chez ces auteurs, nous observions que la mention de la misère comme cause du banditisme répondait à des reconstructions clairement teintées d'ethnocentrisme et, par conséquent, rejetables. Dans le cas d'Appien, bien que cet ethnocentrisme existe 97 , la description tend à nous présenter des faits : fondamentalement militaires et diplomatiques. Or, si nous prenons comme guide le cas de Galba, puisque c'est celui que nous connaissons le mieux, nous observons que le problème de la terre apparaît précisément chez cet auteur. Jusqu'à quel point cela n'est-il pas arrivé dans d'autres cas sur lesquels nous n'avons pas de sources alternatives? Ce soupçon est légitime; néanmoins, afin de nous trouver sur un terrain plus ferme, voyons rapidement quelles ont pu être les étapes successives pour arriver au type de reconstruction que nous lisons chez Appien.

En premier lieu, il faut souligner le changement brutal de paradigme qui se produit entre la période d'expansion maximale de la république romaine, pendant le $\mathrm{II}^{\mathrm{e}}$ siècle et l'instauration de l'empire par Auguste. Si nous donnons des noms d'auteurs aux deux époques, nous voyons que des peuples que Polybe décrit comme spécialement belliqueux sont présentés comme des bandits par Strabon, Tite Live ou Diodore 98. L'idéologie de la pax romana

La Colonizacion Romana y los Planes de Tiberio Graco, Latomus 45, 1986, p. 485-504 et 731-750, recueille la bibliographie la plus récente sur Appien comme historien et met aussi en valeur les problèmes de celui-ci comme source d'étude pour les thèmes coloniaux, mais selon l'analyse qui suit il semble que les problèmes s'étendent à toute la Péninsule.

96. La pertinence de l'inclusion de Tite Live est montrée dans M.R. GIROD, La Géographie de Tite Live, ANRW, II, 30, 2, BerlinNew York 1982, p. 1190-1229, passim.

97. Voir $I b ., 54$ et son analyse supra p. 64-65.

98. Cf. la description des moyens de subsistance dans les Alpes selon Pol., III, 34-35 et 48 avec Str., IV, 6, 3 ; 6-10 et Liv., XXI, 32 s. ; les mêmes différences pour les montagnes proches de la Mer Caspienne dans Pol., V, 44, et Str., XI, 12, 4 et 13, 3 ; la même chose, déjà signalée, se passe dans le traitement de la richesse de la Lusitanie entre Polybe et Strabon, cf. supra. Cf. HARRIS, Guerra e Imperialismo, passim sur le 
"interdit" les guerres, les actions militaires sont décrites comme des actions de police contre des groupes marginaux et non pas comme des campagnes contre des entités sociales et politiques autonomes. En ce sens, Appien, bien qu'il dépende de sources antérieures, nous fournit une reconstruction filtrée indubitablement par l'hégémonie idéologique de la pax romana. Cependant ceci explique seulement pourquoi nous lisons, dans nos textes, des histoires de bandits, mais pas la raison pour laquelle leur situation est provoquée par des causes économiques.

Pour y répondre nous devons considérer qu'entre Polybe et Strabon ou Tite Live il s'est passé beaucoup de choses et que se situe, en particulier, l'œuvre de Poséidonios. Sans prétendre étudier à fond cet auteur-clé, il peut paraître opportun d'examiner la responsabilité qu'il a pu avoir dans l'établissement d'une causalité économique pour le banditisme. En effet, si l'idéologie augustéenne a une influence sur la manière de présenter les faits de guerre à Rome comme actions de police, les causes éventuelles du banditisme échappent au cadre de l'idéologie impériale. De plus, alors que les services de Strabon et Tite Live à la propagande augustéenne sont hors de doute ${ }^{99}$, ce n'est pas le cas de Diodore (contemporain de César) et pourtant il ne laisse pas de refléter clairement le thème de la causalité économique 100.

Si nous tenons compte de tout cela, il semble opportun de penser que Poséidonios est l'auteur de cette explication, il va sans dire que, devant l'état fragmentaire de son œuvre, les arguments sont toujours faibles mais ils sont, dans ce cas, confluents: (a) Diodore le suit apparemment directement dans des passages où sont évoquées la

changement de perception qui s'apprécie dans les sources selon l'époque.

99. Cf. F. LASSERRE, Strabon devant l'Empire Romain, ANRW, II, 30, 1, Berlin-New York 1982, p. 867-896 ; dans p. 881-2 il montre que le récit de la soumission des voleurs des Alpes reproduit presqu'au pied de la lettre des sources augustéennes. Voir aussi, P. THOLLARD, Barbarie et civilisation chez Strabon, Paris 1987, passim, qui essaie de mettre en valeur l'originalité des conceptions anthropologiques de Strabon.

100. Voir V, 34, 6; XXXIII, 1, 4 ; cf. Caes. BG III, 17, 4 ; J.J. TIERNEY, The Celtic Ethnography of Posidonius, Proceedings of the Royal Irish Academy 40, 1960, p. 189-275, p. 211-218 insiste sur la dépendance de César par rapport à Poséidonios. 
misère et la faim de terres comme cause du banditisme 101 ; (b) Strabon dépend de lui dans certains passages-clés que nous avons étudiés dans le même sens 102 ; (c) comme nous l'avons vu, Appien insiste sur la mention de la faim de terres dans la deuxième moitié du II siècle, or ce serait précisément la période que Poséidonios couvre avec le plus de détails dans son œuvre historique 103 ; (d) dans certains textes, nous apprécions un soin particulier dans l'utilisation d'un vocabulaire d'analyse politique, qui est en accord avec des catégories clairement helléniques 104 ; (e) enfin, la dynamique des cités grecques est décrite, dans l'historiographie et la sociologie helléniques, en termes d'affrontements entre riches et pauvres 105. Ainsi, puisque la description des faits de la deuxième moitié du II siècle se présente comme une adaptation de ces catégories et un reflet de ce type d'analyse, nous devons considérer que c'est Poséidonios qui les a introduits pour expliquer les faits de la Péninsule.

S'il en est ainsi, il faudrait peut-être s'en féliciter. On aurait, alors, rempli la condition positiviste de trouver la source la plus proche des événements et on lui octroierait, pour cette raison, le label de la véracité. Néanmoins, cette conclusion me semble précipitée.

101. Cette attribution est discutable: THEILER, Poseidonios, édite D.S. V, 33-38 comme fr. $n^{\circ} 89$ et D.S. XXXIII, 1 , comme fr. $n^{\circ} 96$ a. Pour sa part JACOBY, FGrHis 87 F 117 recueille seulement D.S. V, 33-38. Comme nous l'avons déjà vu, EDELSTEIN et KIDD n'éditent jamais Diodore, voir supra n. 42. Tout dépend des critères suivis pour l'attribution des fragments, $W$. Theiler considère que des passages étendus de Diodore sont de Poséidonios, comme F. Jacoby, les suit MALITZ, Historien, p. 96s. Néanmoins, Edelstein et Kidd rejettent le recours à cette tradition indirecte, de même que M. LAFRANQUE, Poséidonios d'Apamée, Paris 1964, p. 111.

102. Voir supra p. 65 l'étude de III, 3, 5; bien qu'il ne cite pas Poséidonios et que ce passage ne soit pas recueilli par les éditeurs de celui-ci, il semble difficile de lui attribuer une autre source. F. LASSERRE dans son édition de la collection Budé, p. 6 attribue le texte à Poséidonios.

103. LAFRANQUE, Poséidonios, p. 118-22. Cette dernière appréciation ne veut pas dire que Appien dépende directement de Poséidonios, mais simplement que l'information remonte en dernière instance à celui-ci, avec plus ou moins d'intermédiaires.

104. Cf. supra p. 87-91.

105. Voir J.-P. VERNANT, Mito y Sociedad en la Grecia Antigua, Madrid 1982, p. 5-21: La Lucha de Clases; VIDAL-NAQUET, Cazador, p. 189-223: ¿Eran los Esclavos Griegos una Clase Social? y: Reflexiones sobre la Historiografía Griega de la Esclavitud. 
Nous avons vu, précisément dans les textes de Diodore d'inspiration poséidonienne, que se manifestait un grand manque de compréhension pour les réalités institutionnelles et culturelles indigènes. Dans le cas de Galba, Poséidonios, le stoïcien et l'ami de Rutilius Rufus, dut partager les idées de la tradition philosophique, qui, comme nous l'avons montré, était la moins faible; il se peut même que la présentation des faits que nous lisons chez Appien en soit directement issue. En définitive, Poséidonios aurait introduit l'ordre dans le chaos d'une série de campagnes incessantes, épuisantes et, ce qui est pire pour un philosophe, inexplicables : son argumentation consiste en la recherche d'un fondement économique du banditisme, pour cela il transfère des analyses grecques sur les processus de stasis sans les vérifier dans une réalité hispanique que, par ailleurs, il méprise. Pour ma part, si je ne me suis pas trop égaré dans toutes les analyses qui précèdent, je considère que dans l'actualité il n'y a aucune raison d'accepter les thèses de Poséidonios.

\section{Conclusions}

Pour résumer mon article consacré à l'examen de l'historiographie sur la Péninsule, je proposais : (a) le recours au comparatisme, en suivant les pas de J. Costa et en étant conscient des changements qu'il y a eu dans les diverses disciplines impliquées; (b) l'emploi de modèles et de théories anthropologiques sûrs et dont la fécondité est prouvée; (c) l'examen critique des sources. Dans le travail présent, j'ai uniquement abordé systématiquement cette dernière dimension. Le résultat me semble clair dans son aspect négatif : les sources alléguées pour le maintien de la communis opinio en ce qui concerne les peuples péninsulaires protohistoriques au moment de la conquête sont contradictoires, ou peuvent être interprétées autrement, ou requièrent un examen plus poussé. La part positive du travail reste à faire. Je pense qu'elle pourrait suivre deux lignes parallèles dont la confrontation ferait surgir, a posteriori, un nouvel éclairage.

En premier lieu, il faut considérer les peuples préromains dans toute leur spécificité, et non pas en tant que sujets, ennemis ou rebelles à Rome. Certes, en ce sens, les sources disponibles ne sont pas appropriées, mais leurs déformations peuvent être étudiées et "isolées" grâce au comparatisme. Ceci doit être envisagé, à son tour, sous deux angles : l'un correspondant au domaine de "l'anthropologie sociale", où entrent en discussion des peuples avec des caractéris- 
tiques homogènes détectées auparavant et aussi bien antiques que modernes; l'autre aspect se réfère aux réalités idéologiques, en suivant les études de G. Dumézil. Ce travail permettra de rejeter les considérations ethnocentriques en usage sur la barbarie, la sauvagerie, le primitivisme, le manque de culture de ces peuples, qui, en fait, se bornent à reproduire les paradigmes des conquérants et des ethnographes romains ou grecs qui étaient à leur service.

En second lieu, il faut aussi reconnaître, bien que ceci puisse sembler paradoxal, la spécificité de l'action romaine dans la Péninsule, qui n'acquerra un sens plein que si on la compare à celle qui est menée à bien dans d'autres contrées. Ainsi, par exemple, une étude prosopographique des magistrats qui sont intervenus en Hispanie, en considérant leurs carrières antérieures et postérieures, serait très utile. Grâce à celle-ci, nous pourrions évaluer avec précision des actions comme l'affaire Galba, les politiques plus ou moins répressives ou conciliatrices suivies par chacun, etc. Dans ce sens, les travaux en cours sur l'implantation des cadastres dans la Péninsule constituent une autre contribution importante, leur extension à des régions situées hors du Bassin Méditerranéen aurait beaucoup d'intérêt, que les résultats en soient positifs ou négatifs. Dans ces perspectives, le fait que les indigènes se définissent par tel ou tel trait culturel n'a pas d'importance: les magistrats agissent en pensant à leurs carrières romaines et les cadastres effacent les traces des habitats indigènes des différentes régions.

Quoi qu'il en soit, il ne me paraît pas essentiel que l'on soit d'accord avec ces lignes de travail, pour le moment. Mais il me semble fondamental qu'on reconnaisse que les thèmes abordés ici sont ouverts malgré une historiographie qui, à partir des années quarante jusqu'à très récemment, s'était efforcée d'affirmer le contraire. Comme nouvelle tâche positive, il faut faire sauter le verrou intellectuel imposé pendant cette époque et nous rattacher, à notre manière, à l'ouverture d'idées qui caractérisait les œuvres de J. Costa et $P$. Bosch Gimpera, en ajoutant la seule chose qui leur manque : l'examen critique des sources, rendu impossible alors à cause de l'influence intellectuelle allemande reçue à travers un personnage comme A. Schulten 106.

Marco V. GARCIA QUINTELA

106. Je remercie J.J. Segui Marco qui a lu une première version de cet article et qui ne partage pas toutes mes conclusions. Article traduit par M.P. Bouyssou. 


\section{Resumen}

El presente articulo examina sistemáticamente las fuentes aducidas por la communis opinio en favor de sus tesis sobre Hispania en el momento de su conquista, vigentes desde hace un siglo. Del examen se concluye la necesidad de cambiar la aproximación a esas fuentes y de proponer un nuevo modelo para entender esas sociedades y ese período. En efecto, en contra de las ideas admitidas se muestra : que los indígenas tenían unos recursos abundantes y variados ; que los testimonios aducidos en favor de desigualdades sociales no son tan claros como se pretende ; que los generales romanos no son ni "buenos" ni "malos" son, simplemente, generales que tienen más o menos éxito ; que la fundación por Roma de ciudades pobladas por indígenas tiene un componente estrictamente político que se impone sobre el social, único habitualmente considerado.

\section{Résumé}

Cet article examine systématiquement les sources auxquelles la communis opinio fait référence pour soutenir ses thèses sur l'Hispanie au moment de la conquête romaine, thèses qui sont en vigueur depuis un siècle. Leur examen conduit à envisager une nouvelle approche et à proposer un nouveau modèle pour comprendre ces sociétés et cette période. En effet, contrairement aux idées admises, on montre : que les témoignages évoqués pour montrer l'existence d'inégalités sociales ne sont pas aussi convaincants qu'on le prétend ; que les généraux romains ne sont ni "bons" ni "méchants", mais tout simplement des généraux qui ont plus ou moins de succès ; que la fondation par Rome de cités habitées par des indigènes est une mesure surtout politique, et non pas seulement sociale comme on l'affirme habituellement. 